U.S. GEOLOGICAL SURVEY CIRCULAR 1084

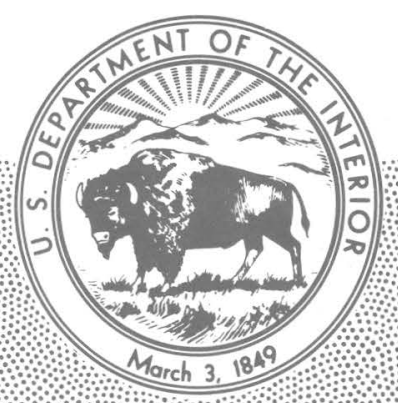

\title{
Catalogue of U.S. Geological Survey Strong-Motion Records, 1989
}




\section{AVAILABILITY OF BOOKS AND MAPS OF THE U.S. GEOLOGICAL SURVEY}

Instructions on ordering publications of the U.S. Geological Survey, along with the last offerings, are given in the current-year issues of the monthly catalog "New Publications of the U.S. Geological Survey." Prices of available U.S. Geological Survey publications released prior to the current year are listed in the most recent annual "Price and Availability List." Publications that are listed in various U.S. Geological Survey catalogs (see back inside cover) but not listed in the most recent annual "Price and Availability List" are no longer available.

Prices of reports released to the open files are given in the listing "U.S. Geological Survey Open-File Reports," updated monthly, which is for sale in microfiche from U.S. Geological Survey Book and Open-File Report Sales, Box 25425, Denver, CO 80225.

Order U.S. Geological Survey publications by mail or over the counter from the offices given below.

\section{BY MAIL}

\section{Books}

Professional Papers, Bulletins, Water-Supply Papers, Techniques of Water-Resources Investigations, Circulars, publications of general interest (such as leaflets, pamphlets, booklets), single copies of periodicals (Earthquakes \& Volcanocs, Preliminary Determination of Epicenters), and some miscellaneous reports, including some of the foregoing series that have gone out of print at the Superintendent of Documents, are obtainable by mail from

\section{U.S. Geological Survey, Book and Open-File Report Sales Box 25425 \\ Denver, CO 80225}

Subscriptions to periodicals (Earthquakes \& Volcanoes and Preliminary Determination of Epicenters) can be obtained ONLY from

\section{Superintendent of Documents \\ U.S. Government Printing Orfice Washington, DC 20402}

(Check or money order must be payable to Supcrintendent of Documents.)

\section{Maps}

For maps, address mail orders to

$$
\begin{gathered}
\text { U.S. Geological Survey, Map Sales } \\
\text { Box } 25286 \\
\text { Denver, CO } 80225
\end{gathered}
$$

Residents of Alaska may order maps from

$$
\begin{aligned}
& \text { U.S. Geological Survey, Map Sales } \\
& 101 \text { Twelfth Ave. - Box } 12 \\
& \text { Fairbanks, AK } 99701
\end{aligned}
$$

\section{OVER THE COUNTER Books}

Books of the U.S. Gcological Survey are available over the counter at the following U.S. Geological Survey offices, all of which are authorized agents of the Superintendent of Documents.

- ANCHORAGE, Alaska--4230 University Dr., Rm. 101

- ANCHORAGE, Alaska--605 West 4th Ave., Rm G-84

- DENVER, Colorado--Federal Bldg., Rm. 169, 1961 Stout St.

- LAKEWOOD, Colorado-- Federal Center, Bldg. 810

- MENLO PARK, California--Bldg. 3, Rm. 3128, 345 Middleficld Rd.

- RESTON, Virginia--National Center, Rm. 1C402, 12201 Sunrise Valley Dr.

- SALT LAKE CITY, Utah--Federal Bldg., Rm. 8105, 125 South State St.

- SAN FRANCISCO, California--Customhouse, Rm. 504, 555 Battery St.

- SPOKANE, Washington--U.S. Courthouse, Rm. 678, West 920 Riverside Ave.

- WASHINGTON, D.C.--U.S. Department of the Interior Bldg., Rm. 2650, 1849 C St., NW.

\section{Maps}

Maps may be purchased over the counter at the U.S. Gcological Survey offices where books are sold (all addresses in above list) and at the following Geological Survey offices:

- ROLLA, Missouri--1400 Independence Rd.

- FAIRBANKS, Alaska--New Federal Buildıng, 101 Twelfth Ave. 


\section{Catalogue of U.S. Geological Survey Strong-Motion Records, 1989}

Compiled by JOSEPHINE C. SWITZER and RONALD L. PORCELLA 


\title{
U.S. DEPARTMENT OF THE INTERIOR MANUEL LUJAN, JR., Secretary
}

\author{
U.S. GEOLOGICAL SURVEY \\ Dallas L. Peck, Director
}

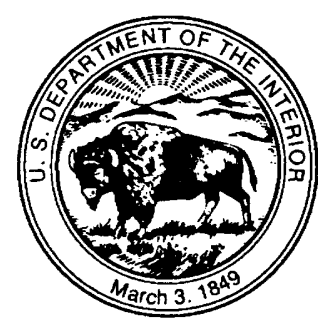

Any use of trade, product, or firm names in this publication is for descriptive purposes only and does not imply endorsement by the U.S. Government

Free on application to

Book and Open-File Report Sales

U.S. Geological Survey

Box 25425

Denver, CO 80225 


\section{PREFACE}

The first seismic engineering program in the United States was administered by the Seismological Field Survey (SFS) of the Coast and Geodetic Survey. This program was begun in 1931 and essentially remained the responsibility of the SFS until 1973, when the U.S. Geological Survey (USGS) assimilated the program into its National Earthquake Hazards Reduction Program. The current Federal seismic engineering program operates the National Cooperative Strong-Motion Network (NCSMN) with more than 1,000 stations in 40 States and Puerto Rico. This network is administered by the USGS in cooperation with both private industry and numerous Federal, State, and local agencies and organizations. Major contributors include the Army Corps of Engineers, the Veterans Administration, and the Metropolitan Water District of Southern California. Primary objectives of the program are to record strong ground motions and the response of representative engineered structures during moderate to large earthquakes, and to disseminate the resultant data and information about the records, sites, and structures to the earthquake engineering research and design community.

This catalogue continues in a revised format the yearly publication "Strong-Motion Program Report, January-December [year]"; it is a continuation of the table 1 summary of accelerograms recovered at NCSMN stations that had been published in that format since 1974. This report includes all accelerograms recovered during 1989. Unless otherwise noted, event data are from the "Preliminary Determination of Epicenters," published monthly by the U.S. Geological Survey. 



\section{CONTENTS}

Preface III

Introduction 1

References 2

TABLE

1. National Cooperative Strong-Motion Network accelerograph records recovered during 19893 



\title{
Catalogue of U.S. Geological Survey Strong-Motion Records, 1989
}

\author{
Compiled by Josephine C. Switzer and Ronald L. Porcella
}

\section{INTRODUCTION}

Nearly 400 accelerograph records were recovered from the National Cooperative Strong-Motion Network (NCSMN) during 1989. Stations in California, Hawaii, and Washington recorded eight earthquakes of $M=5.0$ or greater including the $M_{L}=7.0$ Loma Prieta earthquake in northern California on October 17.

An $\mathrm{M}_{\mathrm{L}}=5.0$ earthquake in the Malibu area of southern California triggered 14 accelerographs at 10 stations on January 19. A peak acceleration of $0.15 \mathrm{~g}$ was recorded at the sixth level of the Wadsworth VA hospital in west Los Angeles (Johnson and Acosta, 1989).

On April 3 an $\mathrm{M}_{\mathrm{L}}=4.7$ earthquake triggered five strong-motion stations along the Calaveras Fault zone in northern California. The peak horizontal acceleration recorded was $0.16 \mathrm{~g}$ at Cherry Flat Reservoir (Salsman and Switzer, 1990).

Two earthquakes on June 12 in southern California, $\mathrm{M}_{\mathrm{L}}=4.4$ and 4.1 , produced records at 13 and 8 NCSMN stations, respectively. Maximum ground accelerations of 0.15 $g$ were recorded in East Los Angeles and at the abutment of Garvey Reservoir in Monterey Park during the 4.4 event; during the 4.1 event, maximum ground motions at these two stations were $0.08 \mathrm{~g}$ and $0.05 \mathrm{~g}$, respectively.

An $\mathrm{M}_{\mathrm{L}}=4.8$ earthquake near Eureka in northern California on September 21 produced significant ground motions at two of five stations triggered by this event; peak motions and their locations were $0.16 \mathrm{~g}$ at Centerville Beach Navy Facility and $0.12 \mathrm{~g}$ at Ferndale Fire Station.

Accelerographs at 41 NCSMN stations in the San Francisco Bay area were triggered by the October 17 Loma Prieta main shock and produced 59 records; these data include recordings from extensively instrumented structures such as high-rise buildings in San Francisco, Berkeley, Hayward, and Emeryville, and a dam east of Morgan Hill. The closest USGS accelerograph station was Anderson Dam, located at an epicentral distance of 27 $\mathrm{km}$, which produced peak accelerations of $0.08,0.23$, and $0.26 \mathrm{~g}$, at the abutment, toe, and downstream stations, respectively (Maley and others, 1989).

A companion project at the USGS has published a report containing the computer processed results of 17 film records and a digital record recovered from this event (Brady and Mork, 1990). A companion tape containing all processed results is available, together with tapes for the remainder of this event's processed records, from the National Geophysical Data Center, 325 Broadway (Mail E/GC1), Boulder, Colorado 80303; phone (303) 497-6084.

An $\mathrm{M}_{\mathrm{L}}=4.2$ earthquake on December 2 triggered seven stations of the Anza strongmotion array in southern California; a peak horizontal ground acceleration of $0.18 \mathrm{~g}$ was recorded on granitic rock at the Keenwild Forest Station site.

Manuscript approved for publication, March 27, 1992 
Additionally, six magnitude 5 or greater earthquakes were recorded at NCSMN stations in 1989. The date, location, magnitude, number of records recovered, and maximum recorded ground motion are as follows: June 26, Hawaii, 6.2, 14 records, $0.19 \mathrm{~g}$; Aug. 8, central California. 5.4, eight records, $0.08 \mathrm{~g}$; Oct. 18, central California, 5.1, three records, less than $0.05 \mathrm{~g}$; Oct. 25, central California, 5.0, two records, less than $0.05 \mathrm{~g}$; Dec. 24, Washington state, 5.1, one record, $0.08 \mathrm{~g}$ (on crest of dam); and Dec. 28, Hawaii, 5.0, two records, less than $0.05 \mathrm{~g}$.

\section{REFERENCES}

Brady, A.G., and Mork, P.N., 1990, Loma Prieta, Califomia, earthquake October 18 (GMT), 1989, processed strong-motion records, Volume I: U.S. Geological Survey Open-File Report 90-247. $274 \mathrm{p}$.

Johnson, D.A., and Acosta, A.V., 1989, Strong-motion data from the Malibu, California, earthquake of January 19, 1989: U.S. Geological Survey Open-File Report 89-186, 21 p.

Maley, R.P., Acosta, A.V., Ellis, F., Etheredge, E.C., Foote, L., Johnson, D.A., Porcella, R.L., Salsman, M., and Switzer, J.C., 1989, U.S. Geological Survey strong-motion records from the northern California (Loma Prieta) earthquake of October 17, 1989: U.S. Geological Survey Open-File Report 89-0568, 85 p.

Salsman, M.J., and Switzer, J.C., 1990, Strong-motion records from earthquakes of June 13, 1988, November 10, 1988, and April 3, 1989, on the Calaveras Fault, central California: U.S. Geological Survey Open-File Report 90-481, 36 p. 
[Station owners: ACOE, U.S. Army Corps of Engineers; BECH, Bechtel Power Corporation; CDOT, California Department of Transportation; CDWR, California Department of Water Resources; JCG, JCG Finance Corporation of America; MWD, Los Angeles Metropolitan Water District; OWNR, Owner of building; UCB, University of California at Berkeley; USGS, U.S. Geological Survey; VA, U.S. Veterans Administration. Instrument trigger time in seconds after the minute or the following minute listed in earthquake column. S-minus trigger denotes $\underline{\mathbf{S}}$-wave-arrival-minus-trigger-time $(\underline{S}-\underline{t})$ or $\underline{\mathbf{S}}$-wave-minus- $\underline{P}$-wave-arrival time $(\underline{S}-\underline{P}$, in brackets) interval. Direction is of case acceleration for upward trace deflection on accelerogram; horizontal components are listed as azimuth, and vertical components as "up" or "down." Maximum amplitude is peak acceleration recorded at ground level on one vertical and two orthogonal horizontal components unless otherwise noted. Duration is interval between first and last peaks of acceleration greater than $0.10 \mathrm{~g}$. Numbers in parentheses refer to footnotes at end of table.]

\begin{tabular}{|c|c|c|c|c|c|c|c|}
\hline Earthquake & $\begin{array}{l}\text { Station Name } \\
\text { (Owner) }\end{array}$ & $\begin{array}{l}\text { Coordinates } \\
\text { (Lat. }{ }^{\circ} \mathrm{N} \\
\text { Long. }{ }^{\circ} \mathrm{W} \text { ) }\end{array}$ & $\begin{array}{l}\text { Trigger } \\
\text { time }\end{array}$ & $\begin{array}{l}\text { S-minus } \\
\text { trigger } \\
\text { (s) }\end{array}$ & $\begin{array}{l}\text { Direction } \\
\quad \text { (az) }\end{array}$ & $\begin{array}{l}\text { Maximum } \\
\text { amplitude } \\
\text { (g) }\end{array}$ & $\begin{array}{l}\text { Duration } \\
\text { (s) }\end{array}$ \\
\hline
\end{tabular}

\begin{tabular}{llrrr}
\hline 9 January 1989 & McGee Creek & 37.550 & (4) & 1.0 \\
0803:26.1 G.m.t. & Mammoth Lakes & 118.811 & & \\
Eastern Calif. & (USGS) SMA-1 & & & \\
37.548N, 118.779W & & 37.550 & (4) & 1.0 \\
Magnitude 3.2 ML & $\begin{array}{l}\text { McGee Creek } \\
\text { Mammoth Lakes }\end{array}$ & 118.811 & & \\
& (USGS) CRA-1 & & &
\end{tabular}

166 m Downhole

35 m Downhole

Surface

$1 \mathrm{~m}$ Downhole

11 January 1989

Salton Sea

33.178

Wildlife Refuge

115.615

$34: 25.6$

1.1

315

(1)

2334:26.5 G.m.t.

(USGS) SMA-1

$33.185 \mathrm{~N}, 115.593 \mathrm{~W}$

Magnitude 3.2 ML

19 January 1989

0653:28.8 G.m.t.

Jensen Filter Plant

34.312

118.496

Balboa Ave.

(MWD)

$33.920 \mathrm{~N}, 118.630 \mathrm{~W}$

Magnitude 5.0 ML

Basement Admin. Bldg.

Generator Bldg.

Reservoir Roof

(1)

Lawndale

33.895

15000 Aviation Blvd.

118.377

(3)

3.5

360
Up
270

.10

.03

(USGS)

.05 
Table 1. National Cooperative Strong-Motion Network Accelerograph Records Recovered During 1989-Continued

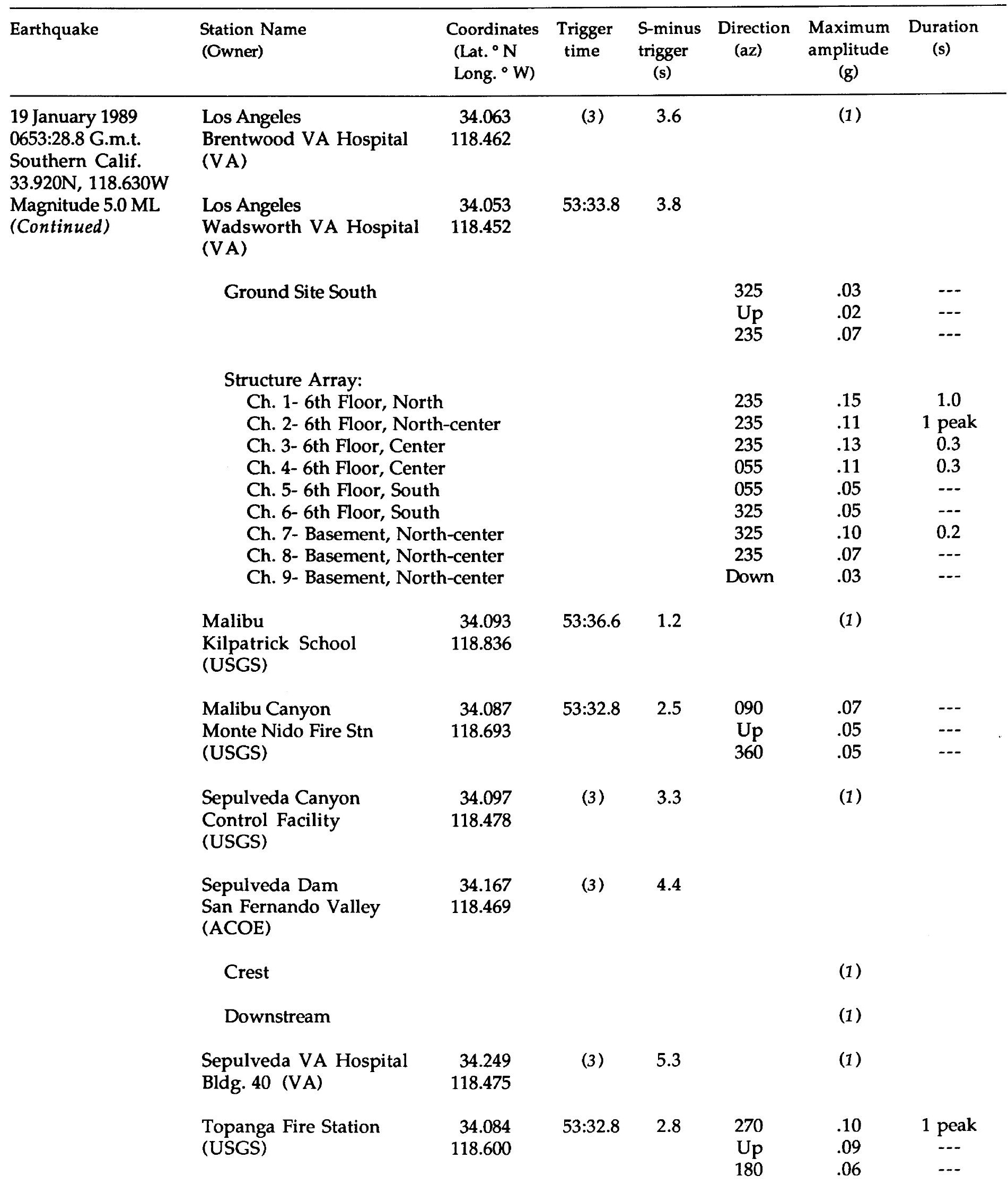


Table 1. National Cooperative Strong-Motion Network Accelerograph Records Recovered During 1989-Continued

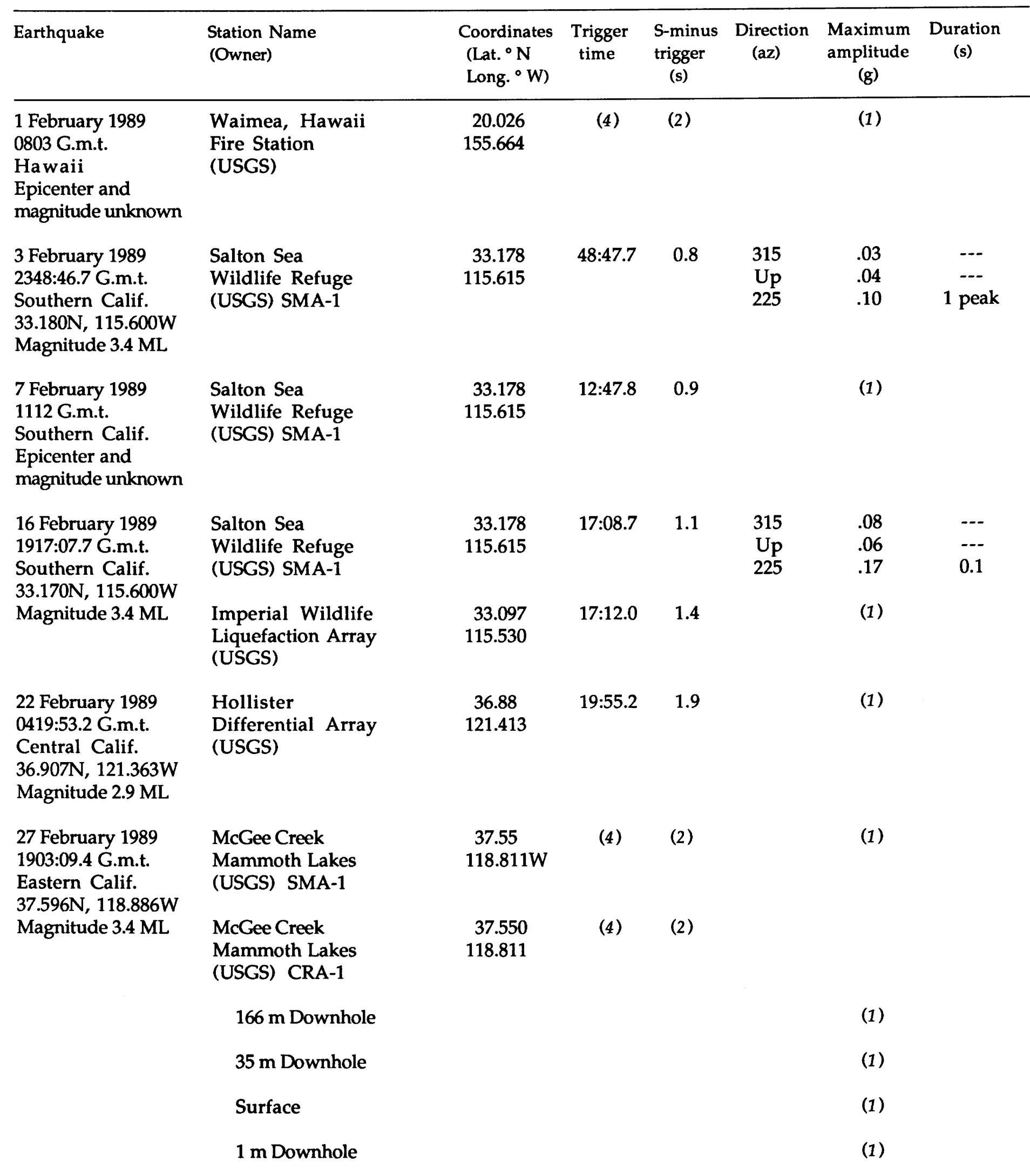


Table 1. National Cooperative Strong-Motion Network Accelerograph Records Recovered During 1989-Continued

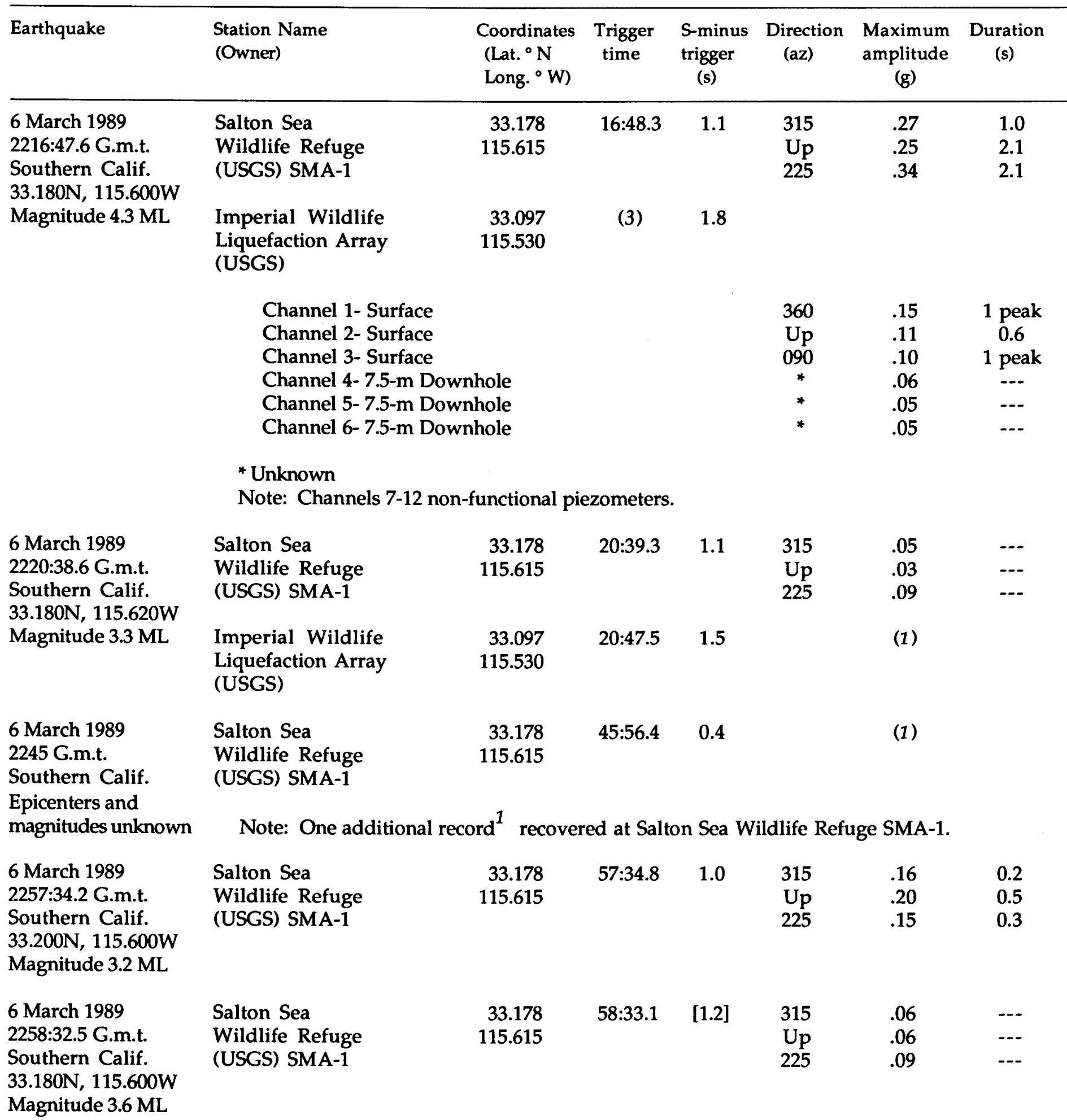


Table 1. National Cooperative Strong-Motion Network Accelerograph Records Recovered During 1989-Continued

\begin{tabular}{|c|c|c|c|c|c|c|c|}
\hline Earthquake & $\begin{array}{l}\text { Station Name } \\
\text { (Owner) }\end{array}$ & $\begin{array}{l}\text { Coordinates } \\
\text { (Lat. }{ }^{\circ} \mathrm{N} \\
\text { Long. }{ }^{\circ} \mathrm{W} \text { ) }\end{array}$ & $\begin{array}{l}\text { Trigger } \\
\text { time }\end{array}$ & $\begin{array}{l}\text { S-minus } \\
\text { trigger } \\
\text { (s) }\end{array}$ & $\begin{array}{l}\text { Direction } \\
\quad(a z)\end{array}$ & $\begin{array}{l}\text { Maximum } \\
\text { amplitude } \\
\text { (g) }\end{array}$ & $\begin{array}{l}\text { Duration } \\
\text { (s) }\end{array}$ \\
\hline $\begin{array}{l}7 \text { March } 1989 \\
\text { 0024:58.1 G.m.t. } \\
\text { Southern Calif. } \\
\text { 33.180N, 115.610W }\end{array}$ & $\begin{array}{l}\text { Imperial Wildlife } \\
\text { Liquefaction Array } \\
\text { (USGS) }\end{array}$ & $\begin{array}{r}33.097 \\
115.530\end{array}$ & $25: 02.5$ & 1.3 & & (1) & \\
\hline Magnitude 4.1 ML & \multicolumn{2}{|c|}{$\begin{array}{l}\text { Channel 1- Surface } \\
\text { Channel 2- Surface } \\
\text { Channel 3- Surface } \\
\text { Channel 4- 7.5-m Downhole } \\
\text { Channel 5- 7.5-m Downhole } \\
\text { Channel 6- 7.5-m Downhole }\end{array}$} & ezometers. & & $\begin{array}{l}360 \\
\mathrm{Up} \\
090 \\
* \\
* \\
*\end{array}$ & $\begin{array}{l}.05 \\
.03 \\
.07 \\
.03 \\
.01 \\
.02\end{array}$ & $\begin{array}{l}\cdots \\
\cdots- \\
\cdots- \\
\cdots- \\
\cdots\end{array}$ \\
\hline $\begin{array}{l}7 \text { March } 1989 \\
\text { 0147:27.5 G.m.t. } \\
\text { Southern Calif. } \\
\text { 33.180N, 115.610W } \\
\text { Magnitude 3.4 ML }\end{array}$ & $\begin{array}{l}\text { Imperial Wildlife } \\
\text { Liquefaction Array } \\
\text { (USGS) }\end{array}$ & $\begin{array}{r}33.097 \\
115.530\end{array}$ & $47: 31.8$ & 1.4 & & (1) & \\
\hline $\begin{array}{l}7 \text { March } 1989 \\
\text { 0743:44.1 G.m.t. } \\
\text { Southern Calif. } \\
\text { 33.180N, 115.590W } \\
\text { Magnitude 4.2 ML }\end{array}$ & $\begin{array}{l}\text { Imperial Wildlife } \\
\text { Liquefaction Array } \\
\text { (USGS) }\end{array}$ & $\begin{array}{r}33.097 \\
115.530\end{array}$ & $43: 48.5$ & 1.3 & & (1) & \\
\hline $\begin{array}{l}27 \text { May } 1988- \\
8 \text { March } 1989 \\
\text { Northern Calif. } \\
\text { Epicenter and }\end{array}$ & $\begin{array}{l}\text { Eel River Valley Array } \\
\text { Centerville Beach } \\
\text { (USGS) }\end{array}$ & $\begin{array}{r}40.563 \\
124.348\end{array}$ & (3) & 4.5 & & (1) & \\
\hline magnitude unknown & $\begin{array}{l}\text { Eel River Valley Array } \\
\text { Loleta Fire Station } \\
\text { (USGS) }\end{array}$ & $\begin{array}{r}40.644 \\
124.219\end{array}$ & (3) & (2) & & (1) & \\
\hline $\begin{array}{l}25 \text { November 1988- } \\
10 \text { March } 1989 \\
\text { Southern Calif. }\end{array}$ & $\begin{array}{l}\text { Calipatria } \\
\text { Fire Station } \\
\text { (USGS) }\end{array}$ & $\begin{array}{r}33.13 \\
115.52\end{array}$ & (3) & 1.5 & $\begin{array}{l}315 \\
\mathrm{Up} \\
225\end{array}$ & $\begin{array}{l}.05 \\
.09 \\
.06\end{array}$ & $\begin{array}{l}--- \\
--- \\
--\end{array}$ \\
\hline
\end{tabular}

Epicenters and magnitudes unknown

10 March 1989

0140:25.4 G.m.t.

Eastern Calif.

$37.525 \mathrm{~N}, 118.874 \mathrm{~W}$

Magnitude 3.3 ML
Note: Two additional records ${ }^{1}$ recovered at Calipatria Fire Station.

$\begin{array}{lr}\text { McGee Creek } & 37.550 \\ \text { Mammoth Lakes } & 118.811 \\ \text { (USGS) SMA-1 }\end{array}$

McGee Creek
Mammoth Lakes

(USGS) SMA-1

Channel 1- Surface

090

.03

01

Channel 5- 7.5-m Downhole

* Unknown

Imperial Wildlife

33.097

$47 \cdot 31.8$

(1)

(USGS)

.06

(2)

(1) 
Table 1. National Cooperative Strong-Motion Network Accelerograph Records Recovered During 1989-Continued

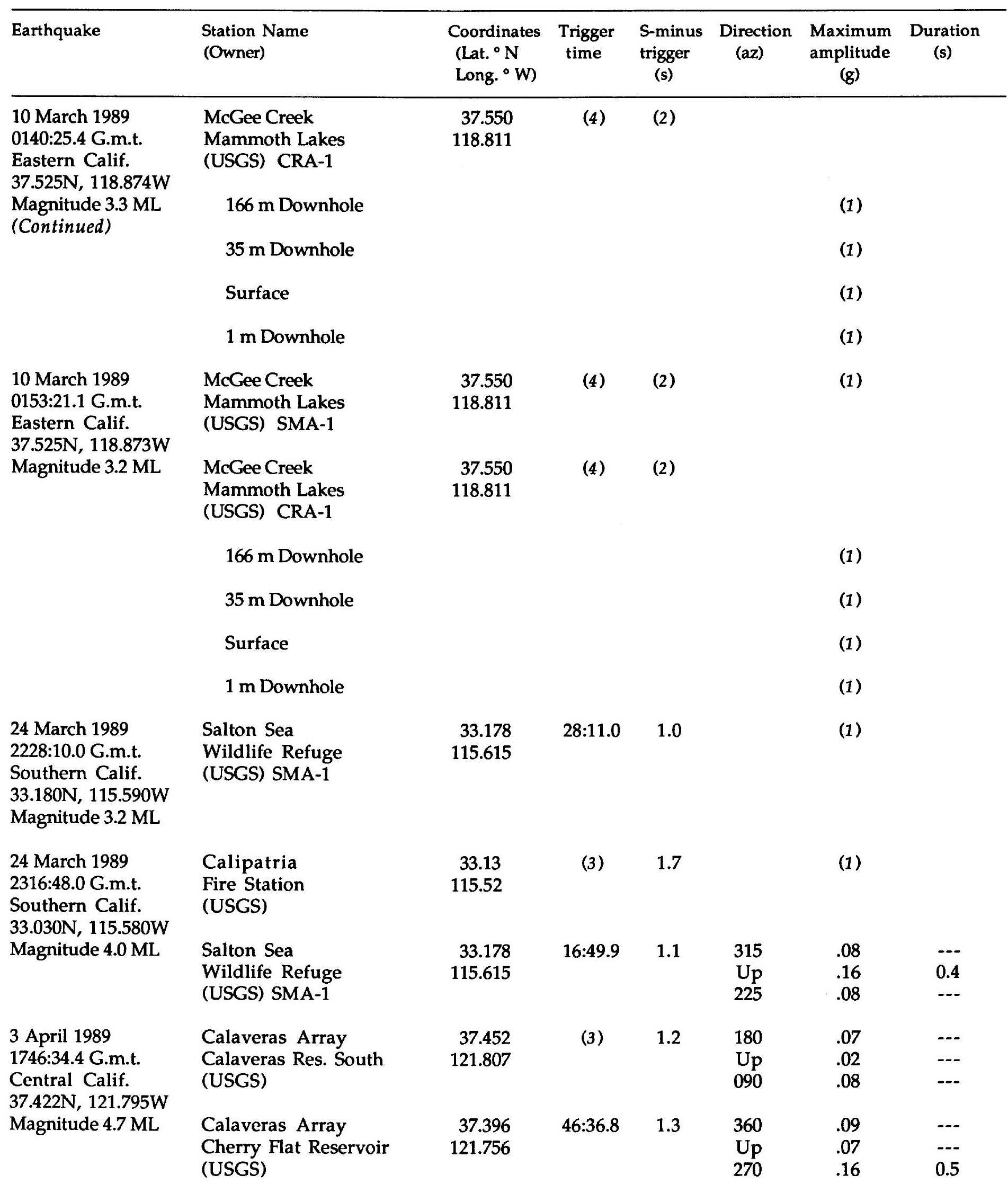


Table 1. National Cooperative Strong-Motion Network Accelerograph Records Recovered During 1989-Continued

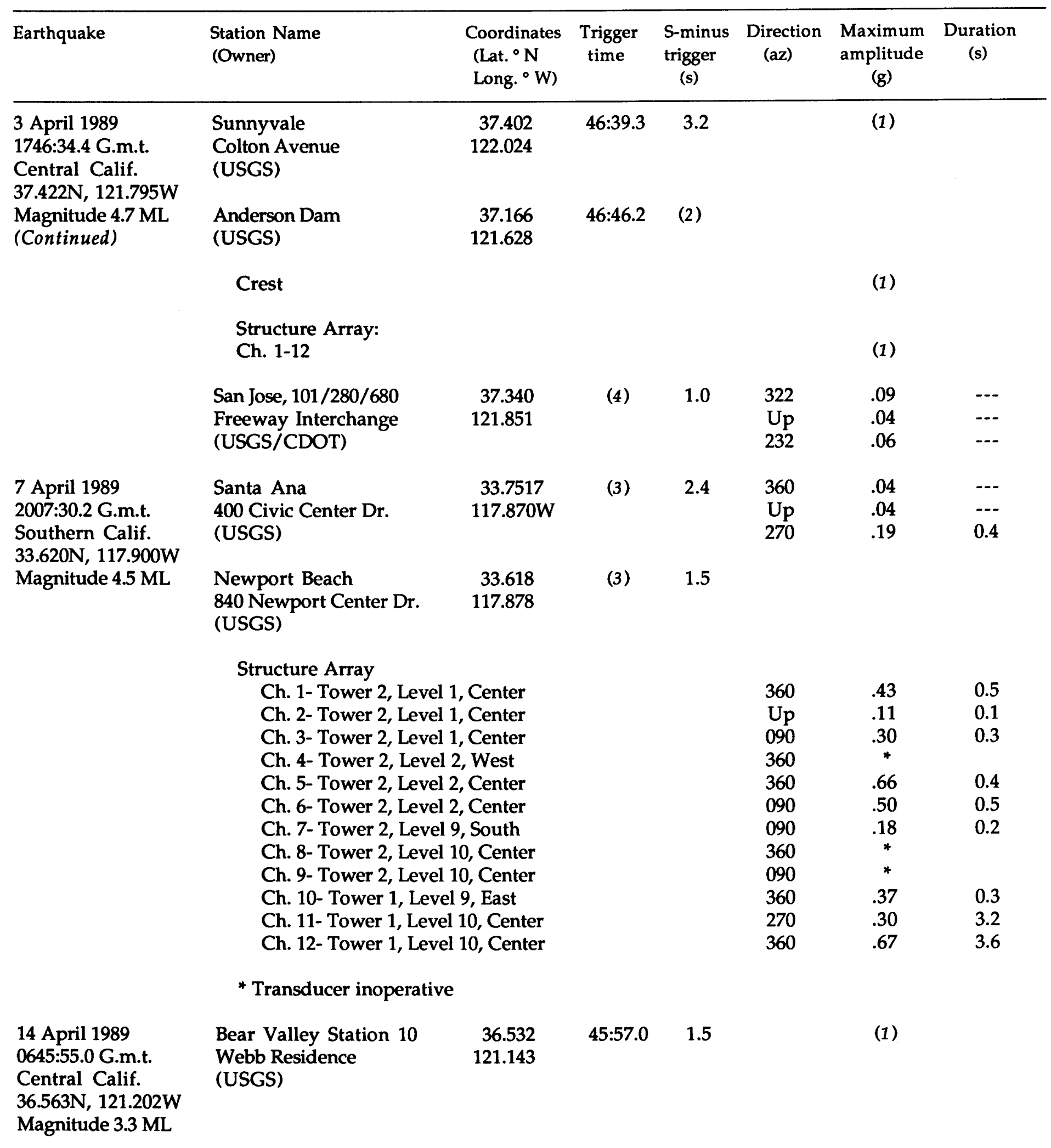


Table 1. National Cooperative Strong-Motion Network Accelerograph Records Recovered During 1989-Continued

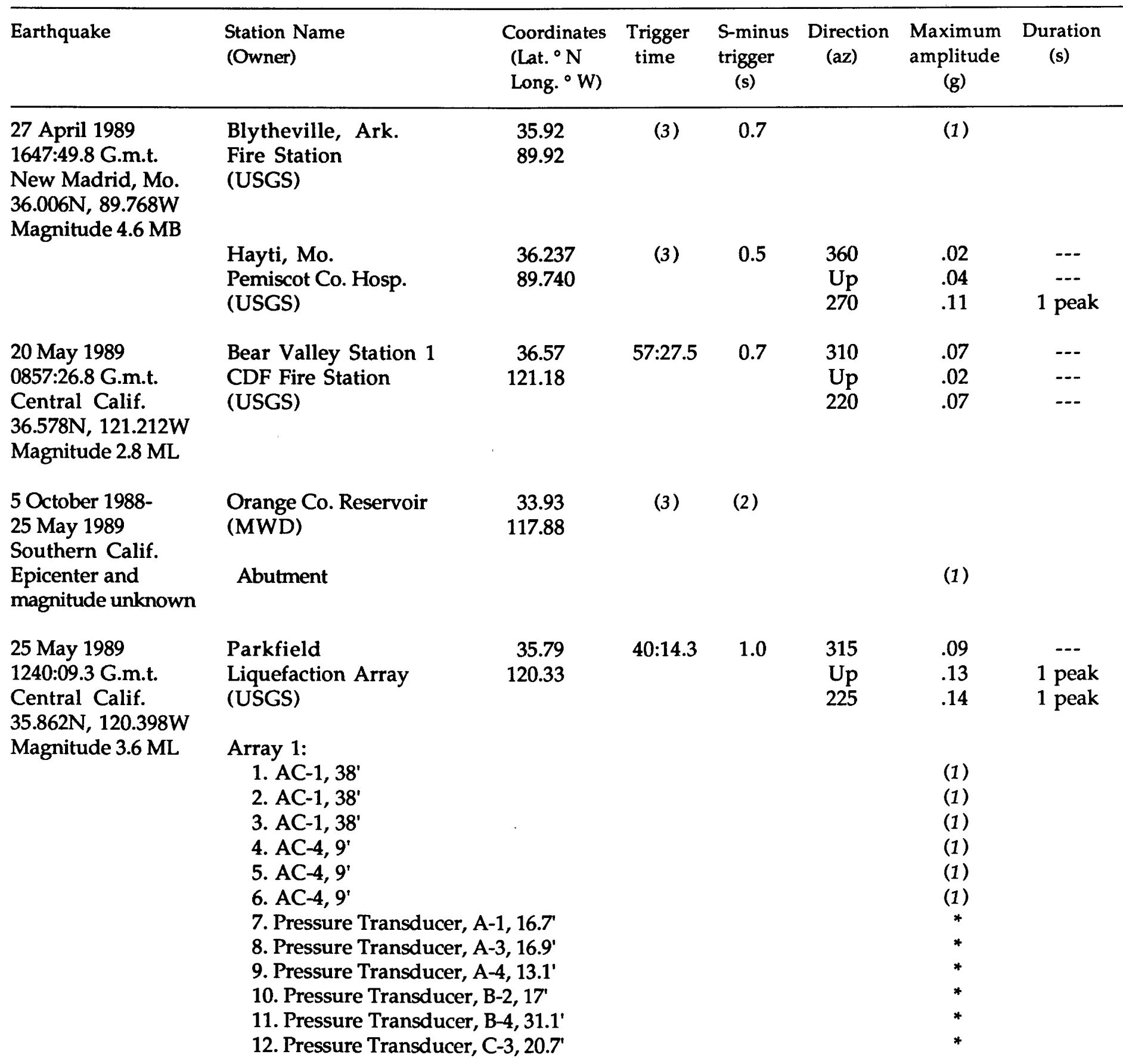


Table 1. National Cooperative Strong-Motion Network Accelerograph Records Recovered During 1989-Continued

\begin{tabular}{|c|c|c|c|c|c|c|c|}
\hline Earthquake & $\begin{array}{l}\text { Station Name } \\
\text { (Owner) }\end{array}$ & $\begin{array}{l}\text { Coordinates } \\
\text { (Lat. }{ }^{\circ} \mathrm{N} \\
\text { Long. }^{\circ} \mathrm{W} \text { ) }\end{array}$ & $\begin{array}{l}\text { Trigger } \\
\text { time }\end{array}$ & $\begin{array}{l}\text { S-minus } \\
\text { trigger } \\
\text { (s) }\end{array}$ & $\begin{array}{l}\text { Direction } \\
\text { (az) }\end{array}$ & $\begin{array}{l}\text { Maximum } \\
\text { amplitude } \\
\text { (g) }\end{array}$ & $\begin{array}{l}\text { Duration } \\
\text { (s) }\end{array}$ \\
\hline
\end{tabular}

25 May 1989

1240:09.3 G.m.t.

Central Calif.

$35.862 \mathrm{~N}, 120.398$

Magnitude 3.6 ML

(Continued)
Array 2:

1. AC-3, 96'

2. AC-3, 96'

3. $\mathrm{AC}-3,96^{\prime}$

4. $\mathrm{AC}-2,13^{\prime}$

5. AC-2, 13'

6. AC-2, 13'

7. Surface

8. Surface

9. Surface

10. Pressure Transducer, D-1, 30.6'

11. Pressure Transducer, D-3, 41.2'

12. Pressure Transducer, $\mathrm{C}-1,20^{\circ}$

* Piezometer trace.

\section{June 1989}

1657:18.4 G.m.t.

Southern Calif. $34.030 \mathrm{~N}, 118.180 \mathrm{~W}$

Magnitude 4.4 ML

34.081
Los Angeles

Jasper St.

(USGS)

118.188

Los Angeles

Bulk Mail Facility

(USGS)

Garvey Reservoir

Monterey Park

(MWD)

Crest

Abutment Bldg.

Los Angeles

1111 Sunset Blvd.

(MWD)

Basement

34.067

118.248

33.996

118.162

34.050

118.114

118.248
(3)

1.1

315

Up

045

130

$\begin{array}{ccc} & (1) & \\ & (1) & \\ & (1) & \\ & (1) & \\ & (1) & \\ & (1) & \\ 315 & .08 & -.- \\ \text { Up } & .08 & -.- \\ 045 & .12 & 1 \text { peak } \\ & * & \\ & * & \\ & * & \end{array}$

Up

.15

.08

.08

(1)

(3) 2.5

(3)

1.6

040

(1)


Table 1. National Cooperative Strong-Motion Network Accelerograph Records Recovered During 1989-Continued

\begin{tabular}{|c|c|c|c|c|c|c|c|}
\hline Earthquake & $\begin{array}{l}\text { Station Name } \\
\text { (Owner) }\end{array}$ & $\begin{array}{l}\text { Coordinates } \\
\text { (Lat. }{ }^{\circ} \mathrm{N} \\
\text { Long. }{ }^{\circ} \mathrm{W} \text { ) }\end{array}$ & $\begin{array}{l}\text { Trigger } \\
\text { time }\end{array}$ & $\begin{array}{l}\text { S-minus } \\
\text { trigger } \\
\text { (s) }\end{array}$ & $\begin{array}{l}\text { Direction } \\
\quad(a z)\end{array}$ & $\begin{array}{l}\text { Maximum } \\
\text { amplitude } \\
\text { (g) }\end{array}$ & $\begin{array}{l}\text { Duration } \\
\text { (s) }\end{array}$ \\
\hline
\end{tabular}

\begin{tabular}{llrll}
\hline 12 June 1989 & Jensen Filter Plant & 34.309 & (3) & 4.0 \\
1657:18.4 G.m.t. & Balboa Ave. & 118.499 & & \\
Southern Calif. & (MWD) & & &
\end{tabular}

34.030N, 118.180W Magnitude 4.4 ML (Continued)

Basement Admin. Bldg.

Generator Bldg.

Reservoir Roof

$\begin{array}{lrrr}\text { Los Angeles } & 34.052 & 57: 21.9 & 2.6 \\ 1100 \text { Wilshire Blvd. } & 118.263 & & \\ \text { (JCG/USGS) } & & & \end{array}$

(1)

(1)

Basement 4 NW

(1)

Basement $3 \mathrm{NE}$

Basement 3 SE

Structure Array:

Ch. 1- 12th Floor, North

Ch. 2- 12th Floor, North

Ch. 3- 12th Floor, South

Ch. 4- 13th Floor, North

Ch. 5- 13th Floor, North

Ch. 6- 13th Floor, South

Ch. 7-32nd Floor, North

Ch. 8- 32nd Floor, North

Ch. 9- 32nd Floor, South

Ch. 10-Ground Floor, North

Ch. 11- Ground Floor, North

Ch. 12- Ground Floor, South

Whittier Narrows Dam $\quad 34.020$

Pico Rivera

118.053

(ACOE)

(3) 0.9

Crest

$\begin{array}{lll}028 & .08 & --- \\ \text { Up } & .05 & --- \\ 298 & .07 & ---\end{array}$

Upstream

$\begin{array}{ll}152 & .08 \\ \mathrm{Up} & .03 \\ 062 & .05\end{array}$


Table 1. National Cooperative Strong-Motion Network Accelerograph Records Recovered During 1989-Continued

\begin{tabular}{|c|c|c|c|c|c|c|c|}
\hline Earthquake & $\begin{array}{l}\text { Station Name } \\
\text { (Owner) }\end{array}$ & $\begin{array}{l}\text { Coordinates } \\
\text { (Lat. }{ }^{\circ} \mathrm{N} \\
\text { Long. }{ }^{\circ} \mathrm{W} \text { ) }\end{array}$ & $\begin{array}{l}\text { Trigger } \\
\text { time }\end{array}$ & $\begin{array}{l}\text { S-minus } \\
\text { trigger } \\
\text { (s) }\end{array}$ & $\begin{array}{l}\text { Direction } \\
\quad(a z)\end{array}$ & $\begin{array}{l}\text { Maximum } \\
\text { amplitude } \\
\text { (g) }\end{array}$ & $\begin{array}{l}\text { Duration } \\
\text { (s) }\end{array}$ \\
\hline \multirow{23}{*}{$\begin{array}{l}\text { 12 June } 1989 \\
\text { 1657:18.4 G.m.t. } \\
\text { Southern Calif. } \\
\text { 34.030N, 118.180W } \\
\text { Magnitude 4.4 ML } \\
\text { (Continued) }\end{array}$} & $\begin{array}{l}\text { Norwalk } \\
12400 \text { Imperial Hwy } \\
\text { (USGS/BECH) }\end{array}$ & $\begin{array}{r}33.92 \\
118.07\end{array}$ & (3) & 0.8 & & & \\
\hline & Basement & & & & & (1) & \\
\hline & 4th Floor & & & & 090 & .02 &.-- \\
\hline & & & & & $\mathrm{Up}$ & .01 & --- \\
\hline & & & & & 360 & .06 & -- \\
\hline & Roof (8th Floor) & & & & 090 & .02 &.-- \\
\hline & & & & & $\mathrm{Up}$ & .02 & --- \\
\hline & & & & & 360 & .06 & --- \\
\hline & South Ground Site & $\begin{array}{r}33.915 \\
118.067\end{array}$ & (3) & (2) & & (1) & \\
\hline & North Ground Site & $\begin{array}{r}33.917 \\
118.067\end{array}$ & (3) & 3.0 & & (1) & \\
\hline & $\begin{array}{l}\text { Prado Dam } \\
\text { Corona } \\
\text { (ACOE) }\end{array}$ & $\begin{array}{r}33.890 \\
117.641\end{array}$ & (3) & (2) & & & \\
\hline & Crest & & & & & (1) & \\
\hline & Downstream & & (3) & 0.3 & 090 & .08 & --- \\
\hline & & & & & Up & .03 & --- \\
\hline & & & & & 360 & .08 & --- \\
\hline & Brea Dam & 33.890 & (3) & 0.8 & & & \\
\hline & $\begin{array}{l}\text { Fullerton } \\
\text { (ACOE) }\end{array}$ & 117.925 & & & & & \\
\hline & Crest & & & & & (1) & \\
\hline & Left Abutment & & & & & (1) & \\
\hline & $\begin{array}{l}\text { Alhambra } \\
900 \text { S. Fremont } \\
\text { (USGS) }\end{array}$ & $\begin{array}{r}34.085 \\
118.149\end{array}$ & (3) & & & & \\
\hline & 12th Floor & & & 2.6 & 090 & .04 & --- \\
\hline & & & & & $\mathrm{Up}$ & .07 & $\ldots$ \\
\hline & & & & & 360 & .07 & -- \\
\hline
\end{tabular}


Table 1. National Cooperative Strong-Motion Network Accelerograph Records Recovered During 1989-Continued

\begin{tabular}{|c|c|c|c|c|c|c|c|}
\hline Earthquake & $\begin{array}{l}\text { Station Name } \\
\text { (Owner) }\end{array}$ & $\begin{array}{l}\text { Coordinates } \\
\text { (Lat. }{ }^{\circ} \mathrm{N} \\
\text { Long. }^{\circ} \mathrm{W} \text { ) }\end{array}$ & $\begin{array}{l}\text { Trigger } \\
\text { time }\end{array}$ & $\begin{array}{l}\text { S-minus } \\
\text { trigger } \\
\text { (s) }\end{array}$ & $\begin{array}{l}\text { Direction } \\
\quad(a z)\end{array}$ & $\begin{array}{l}\text { Maximum } \\
\text { amplitude } \\
\text { (g) }\end{array}$ & $\begin{array}{l}\text { Duration } \\
\text { (s) }\end{array}$ \\
\hline
\end{tabular}

12 June 1989

1657:18.4 G.m.t.

Southern Calif.

$34.030 \mathrm{~N}, 118.180 \mathrm{~W}$

Magnitude 4.4 ML

(Continued)

7

Whittier

7215 Bright Ave.

(USGS)

33.977

118.036

Basement

Carbon Canyon Dam
Brea
(ACOE)
Crest

12 June 1989

1722:25.5 G.m.t.

Southern Calif.

$34.020 \mathrm{~N}, 118.180 \mathrm{~W}$

Magnitude 4.1 ML

\subsection{1}

118.188

33.996

33.914

117.839

\section{Los Angeles \\ 4407 Jasper St. \\ (USGS) \\ Los Angeles \\ Bulk Mail Facility \\ (USGS)}

Garvey Reservoir

Monterey Park

(MWD)

Crest

Abutment Bldg.

118.162

34.050

118.114

(118.114

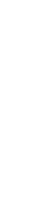

Los Angeles

1111 Sunset Blvd.

(MWD)

Basement

34.067

118.248

(3)

(3) 2.4

(3)

(3)

(2)

180
Up

090

.03
.02
.06

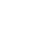


Table 1. National Cooperative Strong-Motion Network Accelerograph Records Recovered During 1989-Continued

\begin{tabular}{|c|c|c|c|c|c|c|c|}
\hline Earthquake & $\begin{array}{l}\text { Station Name } \\
\text { (Owner) }\end{array}$ & $\begin{array}{l}\text { Coordinates } \\
\text { (Lat. }{ }^{\circ} \mathrm{N} \\
\text { Long. }^{\circ} \mathrm{W} \text { ) }\end{array}$ & $\begin{array}{l}\text { Trigger } \\
\text { time }\end{array}$ & $\begin{array}{l}\text { S-minus } \\
\text { trigger } \\
\text { (s) }\end{array}$ & $\begin{array}{l}\text { Direction } \\
\quad \text { (az) }\end{array}$ & $\begin{array}{l}\text { Maximum } \\
\text { amplitude } \\
\text { (g) }\end{array}$ & $\begin{array}{l}\text { Duration } \\
\text { (s) }\end{array}$ \\
\hline
\end{tabular}

\begin{tabular}{llrl}
\hline 12 June 1989 & Jensen Filter Plant & 34.309 & (3) \\
1722:25.5 G.m.t. & Balboa Ave. & 118.499 \\
Southern Calif. & (MWD) & &
\end{tabular}

$34.020 \mathrm{~N}, 118.180$

Magnitude 4.1 ML (Continued)

Administration Bldg.

Basement

Generator Bldg.

Reservoir Roof

(1)

$\begin{array}{lrrr}\text { Los Angeles } & 34.052 & 22: 29.1 & 2.2 \\ 1100 \text { Wilshire Blvd. } & 118.499 & & \\ \text { (JCG/USGS) } & & \end{array}$

Basement 4 NW

(1)

Basement 3 NE

298

Up

208

$\begin{array}{ll}.04 & --- \\ .02 & --- \\ .05 & ---\end{array}$

Basement 3 SE

(1)

Structure Array:

Ch. 1- 12th Floor, North

Ch. 2- 12th Floor, North

Ch. 3- 12th Floor, South

Ch. 4- 13th Floor, North

Ch. 5- 13th Floor, North

Ch. 6- 13th Floor, South

Ch. 7- 32nd Floor, North

Ch. 8- 32nd Floor, North

Ch. 9-32nd Floor, South

Ch. 10- Ground Floor, North

Ch. 11- Ground Floor, North

Ch. 12- Ground Floor, South

Alhambra

900 S. Fremont

(USGS)

12th Floor

34.085

118.149

(3) 2.8

Whittier Narrows Dam

Pico Rivera

(ACOE)

34.031

118.054

Upstream
298

208

208

298

208

208

298

208

208

298

208

208

$\begin{array}{ll}.03 & --- \\ .04 & --- \\ .02 & --- \\ .04 & --- \\ .06 & -.- \\ .02 & --- \\ .01 & --- \\ .02 & --- \\ .01 & --- \\ .03 & --- \\ .03 & --- \\ .04 & ---\end{array}$

(1)

152

Up

062 
Table 1. National Cooperative Strong-Motion Network Accelerograph Records Recovered During 1989-Continued

\begin{tabular}{|c|c|c|c|c|c|c|c|}
\hline Earthquake & $\begin{array}{l}\text { Station Name } \\
\text { (Owner) }\end{array}$ & $\begin{array}{l}\text { Coordinates } \\
\text { (Lat. }{ }^{\circ} \mathrm{N} \\
\text { Long. }{ }^{\circ} \mathrm{W} \text { ) }\end{array}$ & $\begin{array}{l}\text { Trigger } \\
\text { time }\end{array}$ & $\begin{array}{l}\text { S-minus } \\
\text { trigger } \\
\text { (s) }\end{array}$ & $\begin{array}{l}\text { Direction } \\
\quad(a z)\end{array}$ & $\begin{array}{l}\text { Maximum } \\
\text { amplitude } \\
\text { (g) }\end{array}$ & $\begin{array}{l}\text { Duration } \\
\text { (s) }\end{array}$ \\
\hline \multirow{12}{*}{$\begin{array}{l}\text { 26 June } 1989 \\
\text { 0327:03.9 G.m.t. } \\
\text { Hawaii } \\
\text { 19.362N, } 155.083 \mathrm{~W} \\
\text { Magnitude } 6.2 \mathrm{Ms}\end{array}$} & $\begin{array}{l}\text { Hawaii National Park } \\
\text { HVO Admin. Bldg. } \\
\text { (USGS) }\end{array}$ & $\begin{array}{r}19.423 \\
155.291\end{array}$ & (4) & (2) & & (1) & \\
\hline & $\begin{array}{l}\text { Hawaii National Park } \\
\text { HVO Warehouse } \\
\text { (USGS) }\end{array}$ & $\begin{array}{r}19.434 \\
155.264\end{array}$ & (4) & (2) & & (1) & \\
\hline & $\begin{array}{l}\text { Hilo, Hawaii } \\
\text { Hilo Hospital } \\
\text { (USGS) }\end{array}$ & $\begin{array}{r}19.72 \\
155.12\end{array}$ & $(4)$ & 5.7 & $\begin{array}{l}352 \\
\mathrm{Up} \\
262\end{array}$ & $\begin{array}{l}.16 \\
.05 \\
.07\end{array}$ & $\begin{array}{l}0.5 \\
--- \\
---\end{array}$ \\
\hline & $\begin{array}{l}\text { Hilo, Hawaii } \\
\text { Sewage Plant } \\
\text { (USGS) }\end{array}$ & $\begin{array}{r}19.734 \\
155.050\end{array}$ & (4) & (2) & $\begin{array}{l}360 \\
\mathrm{Up} \\
270\end{array}$ & $\begin{array}{l}.07 \\
.03 \\
.05\end{array}$ & $\begin{array}{l}--- \\
--- \\
--\end{array}$ \\
\hline & $\begin{array}{l}\text { Hilo, Hawaii } \\
\text { University of Hawaii } \\
\text { (USGS) }\end{array}$ & $\begin{array}{r}19.707 \\
155.083\end{array}$ & $(4)$ & 5.3 & $\begin{array}{l}360 \\
\text { Up } \\
270\end{array}$ & $\begin{array}{l}.11 \\
.04 \\
.04\end{array}$ & 2 peaks \\
\hline & $\begin{array}{l}\text { Honokaa, Hawaii } \\
\text { Police Station } \\
\text { (USGS) }\end{array}$ & $\begin{array}{r}20.080 \\
155.465\end{array}$ & $(4)$ & (2) & & (1) & \\
\hline & $\begin{array}{l}\text { Honomalino, Hawaii } \\
\text { Macadamia Orchard } \\
\text { (USGS) }\end{array}$ & $\begin{array}{r}19.169 \\
155.169\end{array}$ & $(4)$ & (2) & & (1) & \\
\hline & $\begin{array}{l}\text { Laupahoehoe, Hawaii } \\
\text { Post Office } \\
\text { (USGS) }\end{array}$ & $\begin{array}{r}19.987 \\
155.236\end{array}$ & (4) & (2) & & (1) & \\
\hline & $\begin{array}{l}\text { Mauna Kea, Hawaii } \\
\text { State Park } \\
\text { (USGS) }\end{array}$ & $\begin{array}{r}19.752 \\
155.530\end{array}$ & $(4)$ & (2) & & (1) & \\
\hline & $\begin{array}{l}\text { Mauna Kea Summit } \\
\text { U.K. Observatory } \\
\text { (USGS) }\end{array}$ & $\begin{array}{r}19.826 \\
155.473\end{array}$ & (4) & (2) & & (1) & \\
\hline & $\begin{array}{l}\text { Pahala, Hawaii } \\
\text { Kau Hospital } \\
\text { (USGS) }\end{array}$ & $\begin{array}{r}19.20 \\
155.47\end{array}$ & (4) & (2) & $\begin{array}{l}360 \\
\mathrm{Up} \\
270\end{array}$ & $\begin{array}{l}.05 \\
.02 \\
.05\end{array}$ & $\begin{array}{l}--- \\
--- \\
--\end{array}$ \\
\hline & $\begin{array}{l}\text { Pahoa, Hawaii } \\
\text { Fire Station } \\
\text { (USGS) }\end{array}$ & $\begin{array}{r}19.498 \\
155.951\end{array}$ & (4) & (2) & $\begin{array}{c}087 \\
\text { Up } \\
357\end{array}$ & $\begin{array}{l}.19 \\
.07 \\
.21\end{array}$ & \begin{tabular}{c}
5.1 \\
\hdashline-- \\
4.4
\end{tabular} \\
\hline
\end{tabular}


Table 1. National Cooperative Strong-Motion Network Accelerograph Records Recovered During 1989-Continued

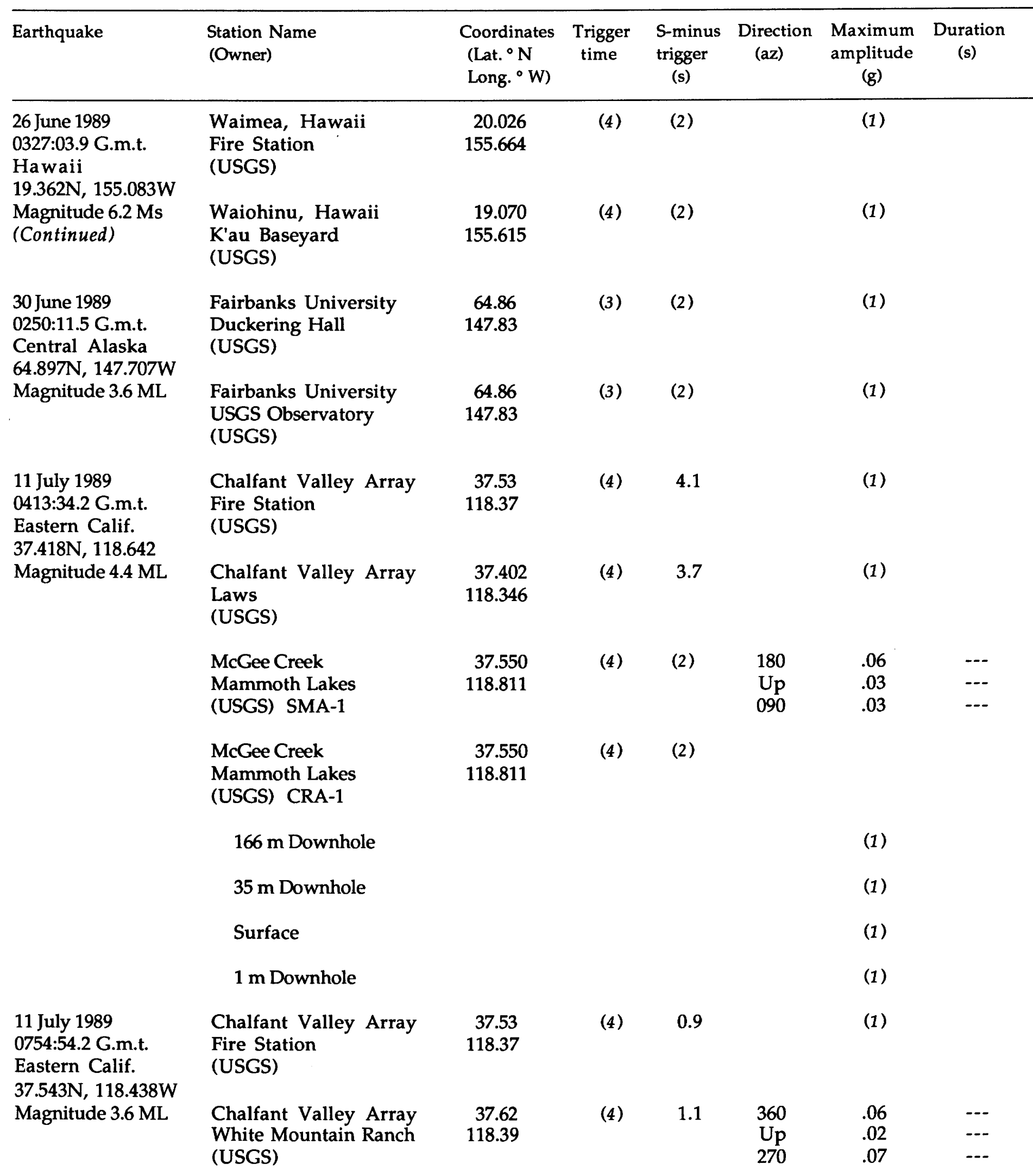


Table 1. National Cooperative Strong-Motion Network Accelerograph Records Recovered During 1989-Continued

\begin{tabular}{|c|c|c|c|c|c|c|c|}
\hline Earthquake & $\begin{array}{l}\text { Station Name } \\
\text { (Owner) }\end{array}$ & $\begin{array}{l}\text { Coordinates } \\
\text { (Lat. }{ }^{\circ} \mathrm{N} \\
\text { Long. }^{\circ} \mathrm{W} \text { ) }\end{array}$ & $\begin{array}{l}\text { Trigger } \\
\text { time }\end{array}$ & $\begin{array}{l}\text { S-minus } \\
\text { trigger } \\
\text { (s) }\end{array}$ & $\begin{array}{l}\text { Direction } \\
\quad \text { (az) }\end{array}$ & $\begin{array}{l}\text { Maximum } \\
\text { amplitude } \\
\text { (g) }\end{array}$ & $\begin{array}{l}\text { Duration } \\
\text { (s) }\end{array}$ \\
\hline
\end{tabular}

\begin{tabular}{llrrr}
\hline 18 July 1989 & Hollister & 36.888 & $07: 24.1$ & 2.0 \\
1107:22.1 G.m.t. & Differential Array & 121.413 & & \\
Central Calif. & (USGS) & & &
\end{tabular}

$36.907 \mathrm{~N}, 121.348 \mathrm{~W}$

Magnitude 3.7 ML

$\begin{array}{llrrr}\text { 18 July } 1989 & \text { Hollister } & 36.888 & 35: 37.9 & 2.0 \\ \text { 1535:36.0 G.m.t. } & \text { Differential Array } & 121.413 & & \\ \text { Central Calif. } & \text { (USGS) } & & & \end{array}$

36.903N, 121.342W

Magnitude 2.8 ML

20 July 1989

0558:44.0 G.m.t.

Central Calif.

36.898N, 121.343W

Magnitude 3.1 ML

2 August 1988-

3 August 1989

Southern Calif.

Epicenters and

magnitudes unknown
Hollister

Differential Array

(USGS)

36.888

121.413

$58: 46.0 \quad 2.0$

34.06

118.29

3000 Leeward Ave.

(OWNR)

Roof (13)

(3)

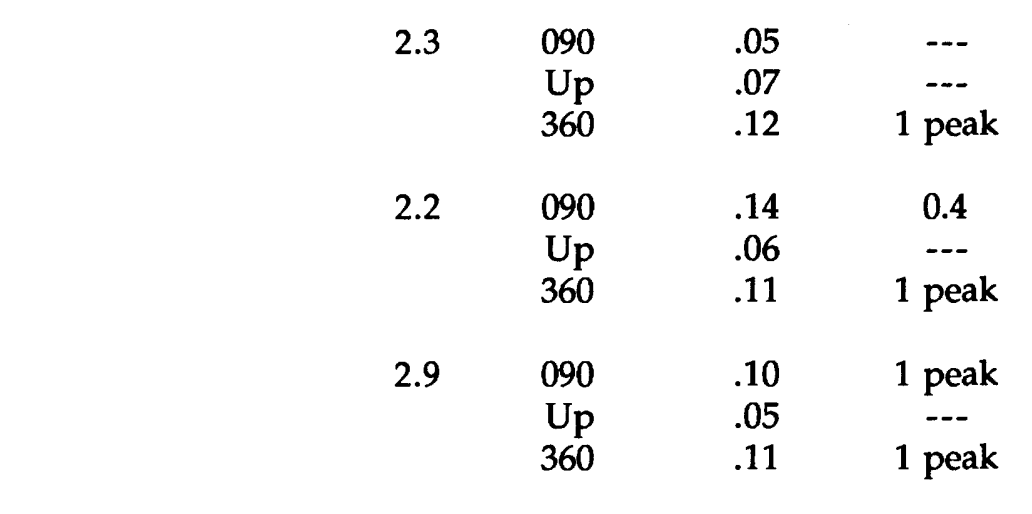

(1)

(1)

(1)

magnitudes unknown

Roof (13)

Note: One additional record ${ }^{1}$ recovered at 3000 Leeward Ave.

18 August 1988-

4 August 1989

Southern Calif.

Epicenters and

magnitudes unknown
Los Angeles

10550 Wilshire Blvd.

(OWNR)

Roof (14)
34.063

118.431

(3)

Note: Two additional records ${ }^{1}$ recovered at 10550 Wilshire Boulevard. 
Table 1. National Cooperative Strong-Motion Network Accelerograph Records Recovered During 1989-Continued

\begin{tabular}{|c|c|c|c|c|c|c|c|}
\hline Earthquake & $\begin{array}{l}\text { Station Name } \\
\text { (Owner) }\end{array}$ & $\begin{array}{l}\text { Coordinates } \\
\text { (Lat. }{ }^{\circ} \mathrm{N} \\
\text { Long. }^{\circ} \mathrm{W} \text { ) }\end{array}$ & $\begin{array}{l}\text { Trigger } \\
\text { time }\end{array}$ & $\begin{array}{l}\text { S-minus } \\
\text { trigger } \\
\text { (s) }\end{array}$ & $\begin{array}{l}\text { Direction } \\
\quad(a z)\end{array}$ & $\begin{array}{l}\text { Maximum } \\
\text { amplitude } \\
\text { (g) }\end{array}$ & $\begin{array}{l}\text { Duration } \\
\text { (s) }\end{array}$ \\
\hline
\end{tabular}

\begin{tabular}{llrrr}
\hline 8 August 1989 & Fremont & 37.535 & (3) & 0.7 \\
0813:27.5 G.m.t. & Emerson Court & 121.929 & & \\
Central Calif. & (USGS) & & & \\
37.130N, 121.952W & & 37.450 & (3) & 4.9 \\
Magnitude 5.4 ML & Milpitas & 121.896 & & \\
& $\begin{array}{l}\text { Rivera St. } \\
\text { (USGS) }\end{array}$ & & & \\
& & & & \\
& Palo Alto & 37.400 & $(3)$ & 4.5 \\
& VA Hospital, Bldg. 1 & 122.140 & & \\
& (VA) & & &
\end{tabular}

Basement

Roof (7th level)

$\begin{array}{lll}302 & .15 & 3.6 \\ U p & .06 & --- \\ 212 & .16 & 0.6\end{array}$

$\begin{array}{lrl}\begin{array}{l}\text { Sunnyvale } \\ \text { Colton Ave. } \\ \text { (USGS) }\end{array} & 37.402 & (3) \\ & 122.024 & \\ \begin{array}{l}\text { Sunnyvale } \\ \text { 1111 Lockheed Way } \\ \text { (USGS) }\end{array} & 37.418 & (3) \\ \begin{array}{l}\text { Stanford University } \\ \text { SLAC Test Lab. } \\ \text { (USGS) }\end{array} & 122.031 & \\ \begin{array}{l}\text { San Francisco } \\ \text { Golden Gate Bridge } \\ \text { (USGS) }\end{array} & 122.205 & \\ \begin{array}{l}\text { Los Angeles } \\ \text { 12121 Wilshire Blvd. } \\ \text { (OWNR) }\end{array} & 122.472 \\ \end{array}$

Events prior to

9 August 1989

Southern Calif.

Epicenters and

magnitudes unknown
Roof (15)
(1)

$\begin{array}{ll}180 & .06 \\ \mathrm{Up} & .01 \\ 090 & .03\end{array}$

(1)

$\begin{array}{ll}360 & .06 \\ \mathrm{Up} & .03 \\ 270 & .05\end{array}$

4.2

090

$\mathrm{Up} \quad .02$

360

.05

360

$\mathrm{Up}$
270

.08

.07

.06

(2)

(1)

.06
.01
.03
(1)


Table 1. National Cooperative Strong-Motion Network Accelerograph Records Recovered During 1989-Continued

\begin{tabular}{|c|c|c|c|c|c|c|c|}
\hline Earthquake & $\begin{array}{l}\text { Station Name } \\
\text { (Owner) }\end{array}$ & $\begin{array}{l}\text { Coordinates } \\
\text { (Lat. }{ }^{\circ} \mathrm{N} \\
\text { Long. }{ }^{\circ} \mathrm{W} \text { ) }\end{array}$ & $\begin{array}{l}\text { Trigger } \\
\text { time }\end{array}$ & $\begin{array}{l}\text { S-minus } \\
\text { trigger } \\
\text { (s) }\end{array}$ & $\begin{array}{l}\text { Direction } \\
\quad \text { (az) }\end{array}$ & $\begin{array}{l}\text { Maximum } \\
\text { amplitude } \\
\text { (g) }\end{array}$ & $\begin{array}{l}\text { Duration } \\
\text { (s) }\end{array}$ \\
\hline $\begin{array}{l}4 \text { June } 1988- \\
21 \text { August } 1989 \\
\text { Hawaii }\end{array}$ & $\begin{array}{l}\text { Mauna Kea, Hawaii } \\
\text { State Park } \\
\text { (USGS) }\end{array}$ & $\begin{array}{r}19.752 \\
155.530\end{array}$ & (3) & (2) & $\begin{array}{l}360 \\
U p \\
270\end{array}$ & $\begin{array}{l}.06 \\
.05 \\
.05\end{array}$ & $\begin{array}{l}--- \\
--- \\
---\end{array}$ \\
\hline
\end{tabular}

Epicenters and magnitudes unknown

Note: One additional record ${ }^{1}$ recovered at Mauna Kea State Park.

\begin{tabular}{|c|c|c|c|c|}
\hline $\begin{array}{l}\text { Mauna Loa, Hawaii } \\
\text { Weather Observatory } \\
\text { (USGS) }\end{array}$ & $\begin{array}{r}19.539 \\
155.580\end{array}$ & (3) & (2) & $\begin{array}{l}360 \\
\text { Up } \\
270\end{array}$ \\
\hline
\end{tabular}

$\begin{array}{llr}2 \text { August 1988- } & \text { Los Angeles } & 34.039 \\ \text { 25 August 1989 } & 1150 \text { S. Hill St. } & 118.259 \\ \text { Southern Calif. } & \text { (OWNR) }\end{array}$

Epicenters and

magnitudes unknown 10th floor

$\begin{array}{rrr}3.5 & 307 & .01 \\ & \text { Up } & .07 \\ 217 & .02\end{array}$

Note: Three additional records ${ }^{1}$ recovered at $1150 \mathrm{~S}$. Hill St.

\begin{tabular}{|c|c|c|c|c|c|c|c|}
\hline \multirow{5}{*}{$\begin{array}{l}21 \text { September } 1989 \\
\text { 1741:18.0 G.m.t. } \\
\text { Northern Calif. } \\
\text { 40.327N, 124.705W } \\
\text { Magnitude 4.8 ML }\end{array}$} & $\begin{array}{l}\text { Eel River Valley Array } \\
\text { Bunker Hill FAA } \\
\text { (USGS) }\end{array}$ & $\begin{array}{r}40.498 \\
124.294\end{array}$ & $41: 26.3$ & 5.7 & & (1) & \\
\hline & $\begin{array}{l}\text { Eel River Valley Array } \\
\text { Centerville Beach } \\
\text { Navy Facility } \\
\text { (USGS) }\end{array}$ & $\begin{array}{r}40.563 \mathrm{~N} \\
134.348 \mathrm{~W}\end{array}$ & $41: 26.6$ & 6.3 & $\begin{array}{l}360 \\
\text { Up } \\
270\end{array}$ & $\begin{array}{l}.16 \\
.03 \\
.09\end{array}$ & 1 peak \\
\hline & $\begin{array}{l}\text { Eel River Valley Array } \\
\text { Ferndale Fire Station } \\
\text { (USGS) }\end{array}$ & $\begin{array}{r}40.58 \\
124.26\end{array}$ & (4) & 7.1 & $\begin{array}{l}360 \\
\mathrm{Up} \\
270\end{array}$ & $\begin{array}{l}.12 \\
.03 \\
.08\end{array}$ & 1 peak \\
\hline & $\begin{array}{l}\text { Eel River Valley Array } \\
\text { Fortuna Fire Station } \\
\text { (USGS) }\end{array}$ & $\begin{array}{r}40.599 \\
124.154\end{array}$ & (3) & 7.8 & $\begin{array}{l}360 \\
\mathrm{Up} \\
270\end{array}$ & $\begin{array}{l}.05 \\
.02 \\
.04\end{array}$ & $\begin{array}{l}--- \\
--- \\
---\end{array}$ \\
\hline & $\begin{array}{l}\text { Eel River Valley Array } \\
\text { Loleta Fire Station } \\
\text { (USGS) }\end{array}$ & $\begin{array}{r}40.644 \\
124.219\end{array}$ & (3) & 7.7 & $\begin{array}{l}360 \\
\mathrm{Up} \\
270\end{array}$ & $\begin{array}{l}.08 \\
.07 \\
.05\end{array}$ & $\begin{array}{l}--- \\
--- \\
---\end{array}$ \\
\hline $\begin{array}{l}30 \text { September } 1989 \\
2349 \text { G.m.t. } \\
\text { Eastern Calif. } \\
\text { Epicenter and } \\
\text { magnitude unknown }\end{array}$ & $\begin{array}{l}\text { Chalfant Valley Array } \\
\text { Laws } \\
\text { (USGS) }\end{array}$ & $\begin{array}{r}37.402 \\
118.346\end{array}$ & (4) & (2) & & (1) & \\
\hline
\end{tabular}


Table 1. National Cooperative Strong-Motion Network Accelerograph Records Recovered During 1989-Continued

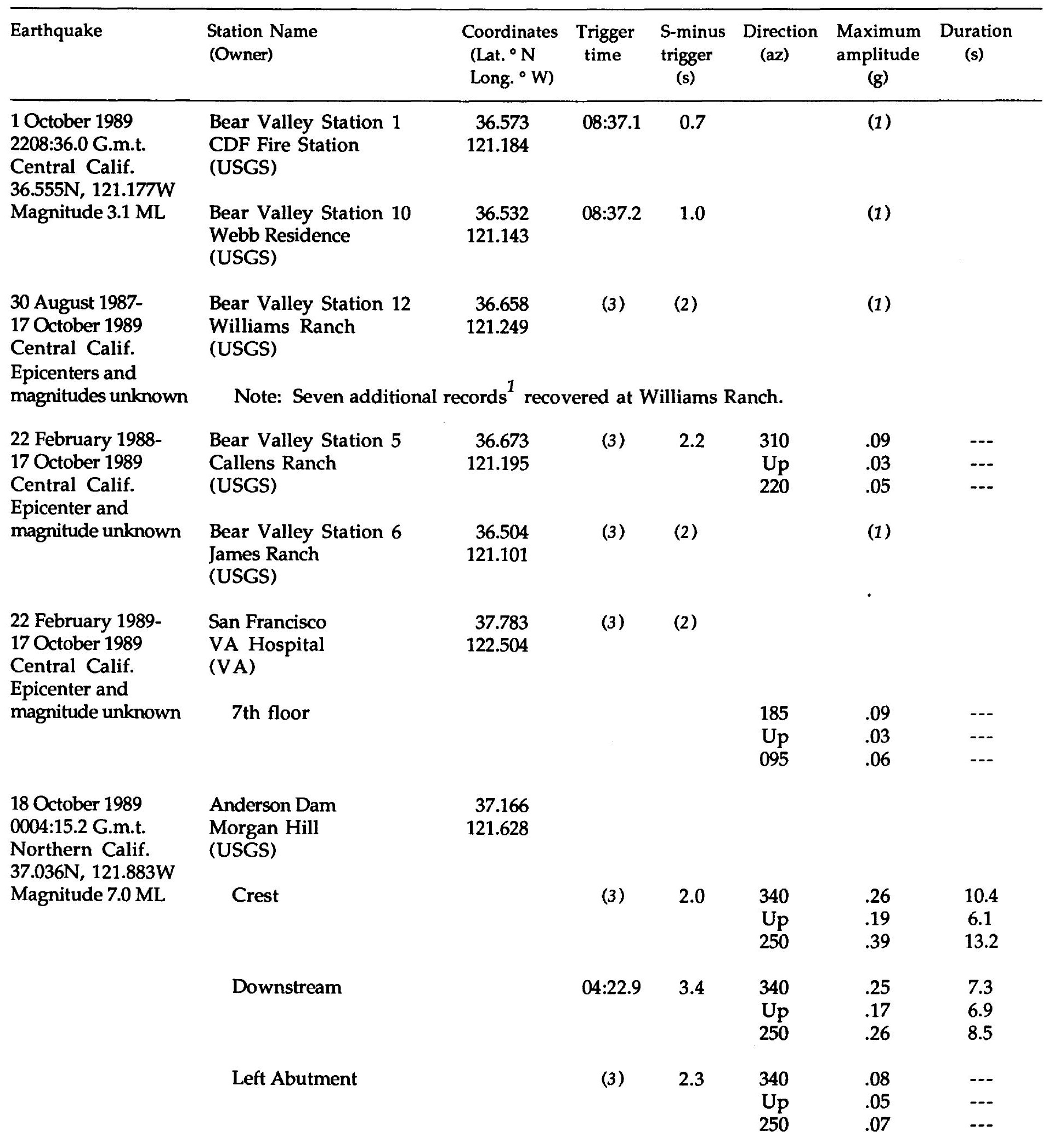


Table 1. National Cooperative Strong-Motion Network Accelerograph Records Recovered During 1989-Continued

\begin{tabular}{|c|c|c|c|c|c|c|c|}
\hline Earthquake & $\begin{array}{l}\text { Station Name } \\
\text { (Owner) }\end{array}$ & $\begin{array}{l}\text { Coordinates } \\
\text { (Lat. }{ }^{\circ} \mathrm{N} \\
\text { Long. }{ }^{\circ} \mathrm{W} \text { ) }\end{array}$ & $\begin{array}{l}\text { Trigger } \\
\text { time }\end{array}$ & $\begin{array}{l}\text { S-minus } \\
\text { trigger } \\
\quad(s)\end{array}$ & $\begin{array}{l}\text { Direction } \\
\quad(a z)\end{array}$ & $\begin{array}{l}\text { Maximum } \\
\text { amplitude } \\
\text { (g) }\end{array}$ & $\begin{array}{l}\text { Duration } \\
\text { (s) }\end{array}$ \\
\hline $\begin{array}{l}18 \text { October } 1989 \\
\text { 0004:15.2 G.m.t. } \\
\text { Northern Calif. } \\
37.036 \mathrm{~N}, 121.883 \mathrm{~W} \\
\text { Magnitude } 7.0 \mathrm{ML} \\
\text { (Continued) }\end{array}$ & $\begin{array}{l}\text { Structure Array: } \\
\text { Ch. 1- Mid-dam, Center } \\
\text { Ch. 2- Mid-dam, Cente } \\
\text { Ch. 3- Mid-dam, Right } \\
\text { Ch. 4- Toe } \\
\text { Ch. 5- Toe } \\
\text { Ch. 6- Toe } \\
\text { Ch. 7- Right Crest } \\
\text { Ch. 8- Right Crest } \\
\text { Ch. 9- Right Crest } \\
\text { Ch. 10- Center Crest } \\
\text { Ch. 11- Center Crest } \\
\text { Ch. 12- Center Crest }\end{array}$ & & (4) & 3.3 & $\begin{array}{c}153 \\
243 \\
063 \\
333 \\
\text { Up } \\
063 \\
333 \\
\text { Up } \\
063 \\
333 \\
\text { Up } \\
063\end{array}$ & $\begin{array}{l}.11 \\
.14 \\
.14 \\
.18 \\
.16 \\
.23 \\
.32 \\
.16 \\
.43 \\
.32 \\
.23 \\
.38\end{array}$ & $\begin{array}{c}5.9 \\
8.8 \\
6.4 \\
4.7 \\
3.9 \\
5.6 \\
11.6 \\
5.6 \\
12.3 \\
10.6 \\
9.6 \\
12.2\end{array}$ \\
\hline & $\begin{array}{l}\text { San Jose, } 101 / 280 / 680 \\
\text { Freeway Interchange } \\
\text { (USGS, CDOT) }\end{array}$ & $\begin{array}{r}37.340 \\
121.851\end{array}$ & (4) & 3.2 & $\begin{array}{l}322 \\
\mathrm{Up} \\
232\end{array}$ & $\begin{array}{l}.18 \\
.08 \\
.13\end{array}$ & $\begin{array}{c}10.5 \\
--- \\
8.9\end{array}$ \\
\hline & $\begin{array}{l}\text { Calaveras Array } \\
\text { Cherry Flat Reservoir } \\
\text { (USGS) }\end{array}$ & $\begin{array}{r}37.396 \\
121.756\end{array}$ & (3) & 5.7 & $\begin{array}{l}360 \\
\mathrm{Up} \\
270\end{array}$ & $\begin{array}{l}.09 \\
.06 \\
.07\end{array}$ & $\begin{array}{l}--- \\
--- \\
---\end{array}$ \\
\hline & $\begin{array}{l}\text { Sunnyvale } \\
\text { Colton Avenue } \\
\text { (USGS) }\end{array}$ & $\begin{array}{r}37.402 \\
122.024\end{array}$ & $04: 24.8$ & 5.7 & $\begin{array}{l}360 \\
\mathrm{Up} \\
270\end{array}$ & $\begin{array}{l}.22 \\
.10 \\
.19\end{array}$ & $\begin{array}{l}9.8 \\
4.8 \\
9.5\end{array}$ \\
\hline & $\begin{array}{l}\text { Hollister Airport } \\
\text { Differential Array } \\
\text { (USGS) }\end{array}$ & $\begin{array}{r}36.888 \\
121.413\end{array}$ & $04: 26.5$ & 5.6 & $\begin{array}{l}255 \\
\mathrm{Up} \\
165\end{array}$ & $\begin{array}{l}.29 \\
.16 \\
.27\end{array}$ & $\begin{array}{l}8.0 \\
3.9 \\
4.6\end{array}$ \\
\hline & $\begin{array}{l}\text { Palo Alto } \\
\text { VA Hospital, Bldg. } 1 \\
\text { (VA) }\end{array}$ & $\begin{array}{r}37.40 \\
122.14\end{array}$ & (3) & 3.5 & & & \\
\hline & Basement & & & & $\begin{array}{l}302 \\
\mathrm{Up} \\
212\end{array}$ & $\begin{array}{l}.34 \\
.20 \\
.38\end{array}$ & $\begin{array}{c}2.2 \\
1.4 \\
5.0\end{array}$ \\
\hline & Roof (7th level) & & & & $\begin{array}{l}302 \\
\text { Up } \\
212\end{array}$ & $\begin{array}{l}1.09 \\
.64 \\
.79\end{array}$ & $\begin{array}{l}13.0 \\
12.0 \\
13.0\end{array}$ \\
\hline & $\begin{array}{l}\text { Hollister } \\
\text { City Hall Annex } \\
\text { Basement (USGS) }\end{array}$ & $\begin{array}{r}36.851 \\
121.402\end{array}$ & $04: 27.5$ & 5.8 & $\begin{array}{l}180 \\
\text { Up } \\
090\end{array}$ & $\begin{array}{l}.23 \\
.22 \\
.25\end{array}$ & $\begin{array}{l}7.7 \\
6.2 \\
6.4\end{array}$ \\
\hline & $\begin{array}{l}\text { Calaveras Array } \\
\text { Calaveras Res. South } \\
\text { (USGS) }\end{array}$ & $\begin{array}{r}37.452 \\
121.807\end{array}$ & (3) & 6.5 & $\begin{array}{l}180 \\
\mathrm{Up} \\
090\end{array}$ & $\begin{array}{l}.13 \\
.07 \\
.08\end{array}$ & $\begin{array}{l}3.5 \\
--- \\
---\end{array}$ \\
\hline
\end{tabular}


Table 1. National Cooperative Strong-Motion Network Accelerograph Records Recovered During 1989-Continued

\begin{tabular}{|c|c|c|c|c|c|c|c|}
\hline Earthquake & $\begin{array}{l}\text { Station Name } \\
\text { (Owner) }\end{array}$ & $\begin{array}{l}\text { Coordinates } \\
\text { (Lat. }{ }^{\circ} \mathrm{N} \\
\text { Long. }{ }^{\circ} \mathrm{W} \text { ) }\end{array}$ & $\begin{array}{l}\text { Trigger } \\
\text { time }\end{array}$ & $\begin{array}{l}\text { S-minus } \\
\text { trigger } \\
\text { (s) }\end{array}$ & $\begin{array}{l}\text { Direction } \\
\quad(a z)\end{array}$ & $\begin{array}{l}\text { Maximum } \\
\text { amplitude } \\
\text { (g) }\end{array}$ & $\begin{array}{l}\text { Duration } \\
\text { (s) }\end{array}$ \\
\hline \multirow{39}{*}{$\begin{array}{l}18 \text { October } 1989 \\
\text { 0004:15.2 G.m.t. } \\
\text { Northern Calif. } \\
37.036 \mathrm{~N}, 121.883 \mathrm{~W} \\
\text { Magnitude } 7.0 \mathrm{ML} \\
\text { (Continued) }\end{array}$} & Hollister & 36.765 & (4) & 3.4 & 360 & .06 &.-- \\
\hline & SAGO Vault & 121.446 & & & Up & .05 & --- \\
\hline & (USGS) & & & & 270 & .04 & --- \\
\hline & & & & & & & \\
\hline & Bear Valley Station 14 & 36.569 & $04: 36.5$ & 7.2 & 310 & .10 & 1 peak \\
\hline & Upper Butts Ranch & 121.431 & & & Up & .08 & $\cdots$ \\
\hline & (USGS) & & & & 220 & .10 & 1 peak \\
\hline & Milpitas & 37.437 & $04: 25.0$ & 4.6 & 360 & .09 & --- \\
\hline & Rivera St. & 121.879 & & & Up & .20 & 2.0 \\
\hline & (USGS) & & & & 270 & .11 & 2 peaks \\
\hline & Stanford University & 37.419 & (4) & 6.2 & 360 & .29 & 6.7 \\
\hline & SLAC Test Lab. & 122.205 & & & $\mathrm{Up}$ & .10 & 2 peaks \\
\hline & (USGS) & & & & 270 & .19 & 2.6 \\
\hline & Menlo Park & 37.468 & (3) & 3.2 & 110 & .12 & 9.5 \\
\hline & VA Hospital, Bldg. 37 & 122.157 & & & Up & .11 & 0.9 \\
\hline & (VA) & & & & 020 & .27 & 3.3 \\
\hline & Fremont & 37.535 & (3) & 7.2 & 180 & .15 & 5.2 \\
\hline & Emerson Court & 121.929 & & & Up & .07 & --- \\
\hline & (USGS) & & & & 090 & .20 & 5.1 \\
\hline & APEEL Array Station 9 & 37.47 & $04: 31.1$ & 4.7 & 227 & .11 & 1 peak \\
\hline & Crystal Springs Res. & 122.32 & & & $\mathrm{Up}$ & .06 & -.- \\
\hline & (USGS) & & & & 137 & .12 & 1 peak \\
\hline & Calaveras Array & 37.597 & (3) & 5.5 & 180 & .07 & --- \\
\hline & Sunol Fire Station & 121.880 & & & Up & .03 & --- \\
\hline & (USGS) & & & & 090 & .10 & 1 peak \\
\hline & APEEL Array Station 2 & 37.52 & (3) & 3.5 & 133 & .23 & 3.2 \\
\hline & Redwood City & 122.25 & & & $\mathrm{Up}$ & .08 & --- \\
\hline & (USGS) & & & & 043 & .28 & 4.4 \\
\hline & Foster City & 37.555 & (3) & 4.8 & 360 & .12 & 3.7 \\
\hline & Menhaden Court & 122.248 & & & $\mathrm{Up}$ & .09 & --- \\
\hline & (USGS) & & & & 270 & .11 & 0.2 \\
\hline & Del Valle Dam & 37.615 & (3) & & & & \\
\hline & (CDWR) & 121.745 & & & & & \\
\hline & Crest & & & 5.4 & 065 & .08 & --- \\
\hline & & & & & $\mathrm{Up}_{\mathrm{p}}$ & .07 & --- \\
\hline & & & & & 335 & .08 & -- \\
\hline & Toe & & & 5.1 & 065 & .06 & --- \\
\hline & & & & & $\mathrm{Up}$ & .03 & --- \\
\hline & & & & & 335 & .04 & -.- \\
\hline
\end{tabular}


Table 1. National Cooperative Strong-Motion Network Accelerograph Records Recovered During 1989-Continued

\begin{tabular}{l} 
Earthquake \\
\hline 18 October 1989 \\
0004:15.2 G.m.t. \\
Northern Calif. \\
37.036N, 121.883W \\
Magnitude 7.0 ML
\end{tabular}

(Continued)

\begin{tabular}{|c|c|c|c|}
\hline $\begin{array}{l}\text { Station Name } \\
\text { (Owner) }\end{array}$ & $\begin{array}{l}\text { Coordinates } \\
\text { (Lat. }{ }^{\circ} \mathrm{N} \\
\text { Long. }{ }^{\circ} \mathrm{W} \text { ) }\end{array}$ & $\begin{array}{c}\text { Trigger } \\
\text { time }\end{array}$ & $\begin{array}{l}\text { S-minu } \\
\text { trigger } \\
\text { (s) }\end{array}$ \\
\hline $\begin{array}{l}\text { Livermore } \\
\text { VA Hospital, Bldg. } 62 \\
\text { (VA) }\end{array}$ & $\begin{array}{r}37.625 \\
121.762\end{array}$ & (3) & 6.7 \\
\hline
\end{tabular}

Basement

Roof (7th)

Bear Valley Station $12 \quad 36.658$

Williams Ranch

(USGS)

121.249

APEEL Array Station 2E

Hayward, Muir School

(USGS)

Bear Valley Station 5

Callens Ranch

(USGS)

Bear Valley Station 1

CDF Fire Station

(USGS)

37.66

122.08

Hayward City Hall

(USGS)
37.679

122.082

36.673

121.195

36.573

121.184

Ground Floor
Ground Site North

Ground Site South

Ground Site North

(3)

9.3

31

Up

220

054

Up

324

04:33.0

6.6

310

Up

220

$04: 36.5$

\section{3}

310

Up

220
Duration

(s)

(g)

(g)

(g)


Table 1. National Cooperative Strong-Motion Network Accelerograph Records Recovered During 1989-Continued

\begin{tabular}{lcc}
\hline Earthquake & $\begin{array}{l}\text { Station Name } \\
\text { (Owner) }\end{array}$ & $\begin{array}{r}\text { Coordi } \\
\text { (Lat. } \\
\text { Long. }\end{array}$ \\
\hline 18 October 1989 & Structure Array: \\
0004:15.2 G.m.t. & Ch. 1- 12th Floor, West \\
Northern Calif. & Ch. 2- 12th Floor, Center \\
37.036N, 121.883W & Ch. 3- 12th Floor, Center \\
Magnitude 7.0 ML & Ch. 4- 7th Floor, West \\
(Continued) & Ch. 5- 7th Floor, Center \\
& Ch. 6- 7th Floor, Center \\
& Ch. 7- 3rd Floor, West \\
& Ch. 8- 3rd Floor, Center \\
& Ch. 9- 3rd Floor, Center \\
& Ch. 10-3rd Floor, Southwest \\
& Ch. 11-3rd Floor, Southwest \\
& Ch. 12- Ground Floor, West \\
&
\end{tabular}

$\begin{array}{lr}\text { Calaveras Array } & 37.709 \\ \begin{array}{l}\text { Dublin Fire Station } \\ \text { (USGS) }\end{array} & 121.932\end{array}$

Bear Valley Station 10

Webb Residence

(USGS)

Bear Valley Station 7

Pinnacles Nat'l Mon.

(USGS)

San Francisco

1295 Shafter St.

(USGS)

San Francisco State U. $\quad 37.724$

Thornton Hall

(USGS)

San Francisco

575 Market St.

(USGS)
122.475

36.532
121.143

36.483
121.180

37.728
122.385


37.724
122.475

37.79

122.40
(3)

6.8

trigger

(s)
Direction Maximum

(az) amplitude

(g)
Duration

(s)

(a)

(a)

$\begin{array}{ccc}334 & .10 & 4.2 \\ 334 & .10 & 1 \text { peak } \\ 064 & .13 & 4.5 \\ 334 & .09 & --- \\ 334 & .08 & --- \\ 064 & .09 & --- \\ 334 & \text { Inoperative } & \\ 334 & .07 & --- \\ 064 & .08 & -.- \\ \text { Up } & .05 & --- \\ \text { Up } & .04 & --- \\ 334 & .07 & ---\end{array}$

Basement

135
Up
045

$\begin{array}{lc}.08 & -- \\ .06 & -- \\ .11 & 1 \text { peak }\end{array}$

1 peak

--.

3.6

$\begin{array}{cccc}04: 36.4 & 5.1 & 310 & .04 \\ & & \text { Up } & .03 \\ & & 220 & .06\end{array}$

.11

.05

.07

(4)

8.8

270

.14

.04

.11

(3) 8.5

3.3
---
0.9

peak

---

$--$

3.3

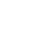


Table 1. National Cooperative Strong-Motion Network Accelerograph Records Recovered During 1989-Continued

\begin{tabular}{lcc}
\hline Earthquake & $\begin{array}{l}\text { Station Name } \\
\text { (Owner) }\end{array}$ & $\begin{array}{c}\text { Coordi } \\
\text { (Lat. } \\
\text { Long. }\end{array}$ \\
\hline 18 October 1989 & Structure Array: \\
0004:15.2 G.m.t. & Ch. 1- 42nd Level, Northwest \\
Northern Calif. & Ch. 2- 42nd Level, Center \\
37.036N, 121.883W & Ch. 3- 42nd Level, Center \\
Magnitude 7.0 ML & Ch. 4- 34th Level, Northwest \\
(Continued) & Ch. 5- 34th Level, Center \\
& Ch. 6- 34th Level, Center \\
& Ch. 7- 25th Level, Northwest \\
& Ch. 8- 25th Level, Center \\
& Ch. 9- 25th Level, Center \\
& Ch. 10- Ground Level \\
& Ch. 11- Ground Level
\end{tabular}

$\begin{array}{lr}\text { San Francisco } & 37.80 \\ \text { 600 Montgomery St. } & 122.40 \\ \text { (USGS) } & \end{array}$

261

045

225

135

045

225

135

045

225

135

045

315

Maximum

mplitude

Duration

(s)

(g)

(s)

6.4

.22

6.9

.14

2.3

.15

6.9

7.0

5.0

.19

.19

.23

.16

.12

.13

6.6

7.0

4.1

1.4

3.9

(4)

8.7
Basement
Basement
29th Floor
49th Floor
Structure Array:
Ch. 2- 21st Floor, South Central
Ch. 3- 21st Floor, South Central
Ch. 4- 5th Floor, West Central
Ch. 5- 5th Floor, South Central
Ch. 6- 5th Floor, South Central
Ch. 7-SE Corner, Foundation
Ch. 8- Ground Level, West Central
Ch. 9- Ground Level, Center
Ch. 10- Ground Level, Center
Ch. 11- Foundation, NW Corner
Ch. 12- Foundation, West Side
Ch. 13- Foundation, SW Corner

Up

171

.12

.05

.11

0.9

$---$

1.2

261

.15

4.5

Up

.11

171

.17

2.1

5.1

261

.31

Up

.14

6.4

171

.29

5.8

351

.20

351

.17

7.1

081

.22

3.0

351

.27

11.8

351

.28

7.2

081

.24

7.1

Up

351

351

081

351

351

Up

4.6

-.-

Emeryville

6363 Christie Ave.

(USGS)

37.844

122.295

(3)

9.2

Ground Site South

5.2

$---$

5.1 
Table 1. National Cooperative Strong-Motion Network Accelerograph Records Recovered During 1989-Continued

\begin{tabular}{|c|c|c|c|c|c|c|c|}
\hline Earthquake & $\begin{array}{l}\text { Station Name } \\
\text { (Owner) }\end{array}$ & $\begin{array}{l}\text { Coordinates } \\
\left(\text { Lat. }^{\circ} \mathrm{N}\right. \\
\text { Long. }{ }^{\circ} \mathrm{W} \text { ) }\end{array}$ & $\begin{array}{l}\text { Trigger } \\
\text { time }\end{array}$ & $\begin{array}{l}\text { S-minus } \\
\text { trigger } \\
\text { (s) }\end{array}$ & $\begin{array}{l}\text { Direction } \\
\text { (az) }\end{array}$ & $\begin{array}{c}\text { Maximum } \\
\text { amplitude } \\
\text { (g) }\end{array}$ & $\begin{array}{l}\text { Duration } \\
\text { (s) }\end{array}$ \\
\hline
\end{tabular}

18 October 1989

0004:15.2 G.m.t.

Northern Calif. 37.036N, 121.883W

Magnitude 7.0 ML

(Continued)

\section{Structure Array 1:}

Ch. 1- Roof (31st), West Wing

Ch. 2- Roof (31st), South Wing

Ch. 3- Roof (31st), North Wing

Ch. 4- Roof (31st), Central Core

Ch. 5- Roof (31st), Central Core

Ch. 6- 21st Floor, Central Core

Ch. 7- 21st Floor, West Wing

Ch. 8- 21st Floor, South Wing

Ch. 9- 21st Floor, North Wing

Ch. 10- 13th Floor, Central Core

Ch. 11- 13th Floor, Central Core

Ch. 12- 21st Floor, Central Core

Structure Array 2:

Ch. 1- 13th Floor, West Wing

Ch. 2- 13th Floor, South Wing

Ch. 3- 13th Floor, North Wing

Ch. 4- Ground Floor, West Wing

Ch. 5- Ground Floor, South Wing

Ch. 6- Ground Floor, Central Core

Ch. 7- Ground Floor, North Wing

Ch. 8- Ground Floor, North Wing

Ch. 9- Ground Floor, North Wing

Ch. 10- Ground Site, North

Ch. 11- Ground Site, North

Ch. 12- Ground Site, North

Berkeley, U.C.

Strawberry Cyn.

37.87

(UCB)

122.24

Berkeley, U.C.

37.87

Haviland Hall

122.26

Basement (UCB)

Berkeley

2168 Shattuck Ave. (USGS)

Basement, East

Basement, West
350

050

290

350

260

350

350

050

290

350

260

260

350

050

290

Up

Up

Up

260

Up

350

350

Up

260

04:38.6

9.8

135

Up

045

.27

.31

.39

.25

.38

.20

.19

.18

.24

.27

.26

.23

9.2

11.1

19.8

11.4

16.5

4.9

1.7

3.7

6.0

5.4

7.0

5.9

4.4

4.8

7.4

-.-

-.-

---

1.9

...

4.5

1.7

$---$

2.7

.22

135

Up

045

.04

.02

.08

$04: 48.2 \quad$ (2)

.03

.02

.06
04:38.3 $6.9 \quad 261$

Up

171

04:38.2
6.8
261

171
.09

.02

.11

1 peak

.10

.03

.09

1 peak

-.- 
Table 1. National Cooperative Strong-Motion Network Accelerograph Records Recovered During 1989-Continued

\begin{tabular}{|c|c|c|}
\hline Earthquake & $\begin{array}{l}\text { Station Name } \\
\text { (Owner) }\end{array}$ & $\begin{array}{l}\text { Coordinate } \\
\text { (Lat. }{ }^{\circ} \mathrm{N} \\
\text { Long. }{ }^{\circ} \mathrm{W}\end{array}$ \\
\hline $\begin{array}{l}18 \text { October } 1989 \\
0004: 15.2 \text { G.m.t. } \\
\text { Northern Calif. } \\
37.036 \mathrm{~N}, 121.883 \mathrm{~W} \\
\text { Magnitude } 7.0 \mathrm{ML} \\
\text { (Continued) }\end{array}$ & \multicolumn{2}{|c|}{$\begin{array}{l}\text { Structure Array: } \\
\text { Ch. 1- 13th Floor, East Core } \\
\text { Ch. 2- 13th Floor, East Core } \\
\text { Ch. 3- 13th Floor, Center } \\
\text { Ch. 4- 13th Floor, Roof West Core } \\
\text { Ch. 5- 13th Floor, Roof West Core } \\
\text { Ch. 6- 13th Floor, Southwest } \\
\text { Ch. 7- 13th Floor, Southwest } \\
\text { Ch. 8- 4th Floor, Southwest } \\
\text { Ch. 9- 4th Floor, Southwest } \\
\text { Ch. 10- 4th Floor, West Core } \\
\text { Ch. 11- 4th Floor, West Core } \\
\text { Ch. 12- 4th Floor, East Core }\end{array}$} \\
\hline
\end{tabular}

San Francisco

37.783

VA Hospital

(VA)

122.504

Basement

7th Floor

San Francisco

Golden Gate Bridge

Abutment (USGS)

Richmond Bulk Mail

2501 Rydin Road

(USGS)

Martinez

VA Hospital

Basement (VA)

Larkspur

Ferry Terminal

(USGS)

37.993

122.115

37.946

122.508

$\begin{array}{ccccc}\begin{array}{c}\text { Trigger } \\ \text { time }\end{array} & \begin{array}{l}\text { S-minus } \\ \text { trigger } \\ (\mathbf{s})\end{array} & \begin{array}{c}\text { Direction } \\ \text { (az) }\end{array} & \begin{array}{c}\text { Maximum } \\ \text { amplitude } \\ (\mathrm{g})\end{array} & \begin{array}{c}\text { Duration } \\ \text { (s) }\end{array} \\ & & & \text { ( }) & \end{array}$

171

261

171

171

081

081

171

171

081

081

171

171

(3)

(2)

.13

1.1

.23

.13

.19

.21

.23

.16

.23

.11

.08

.11

.08

185

Up

.08

.05

.16

-.-

1.8

185

.34

14.5

Up

.08

.22

-.-

5.6

(4)

8.5

360

.12

.06

.24

3.1

$---$

2.9

(4)

$\begin{array}{rr}7.7 \quad 057 \\ \quad \text { Up } \\ & 327\end{array}$

.08

.04

.11

-.-

$---$

0.3

(3)

$8.9 \quad 020$

Up

.07

.03

.05

(4)

9.1

360

Up

.10

.06

270 
Table 1. National Cooperative Strong-Motion Network Accelerograph Records Recovered During 1989-Continued

\begin{tabular}{|c|c|c|c|c|c|c|c|}
\hline Earthquake & $\begin{array}{l}\text { Station Name } \\
\text { (Owner) }\end{array}$ & $\begin{array}{l}\text { Coordinates } \\
\text { (Lat. }{ }^{\circ} \mathrm{N} \\
\text { Long. }^{\circ} \mathrm{W} \text { ) }\end{array}$ & $\begin{array}{l}\text { Trigger } \\
\text { time }\end{array}$ & $\begin{array}{l}\text { S-minus } \\
\text { trigger } \\
\text { (s) }\end{array}$ & $\begin{array}{l}\text { Direction } \\
\quad \text { (az) }\end{array}$ & $\begin{array}{l}\text { Maximum } \\
\text { amplitude } \\
\text { (g) }\end{array}$ & $\begin{array}{l}\text { Duration } \\
\text { (s) }\end{array}$ \\
\hline $\begin{array}{l}18 \text { October } 1989 \\
0007 \text { G.m.t. } \\
\text { Central Calif. } \\
\text { Epicenter and } \\
\text { magnitude unknown }\end{array}$ & $\begin{array}{l}\text { Structure Array: } \\
\text { Ch. 1- Mid-dam, Center } \\
\text { Ch. 2- Mid-dam, Center } \\
\text { Ch. 3- Mid-dam, Right } \\
\text { Ch. 4- Toe } \\
\text { Ch. 5- Toe } \\
\text { Ch. 6- Toe } \\
\text { Ch. 7- Right Crest } \\
\text { Ch. 8- Right Crest } \\
\text { Ch. 9- Right Crest } \\
\text { Ch. 10- Center Crest } \\
\text { Ch. 11- Center Crest } \\
\text { Ch. 12- Center Crest }\end{array}$ & $\begin{array}{r}37.166 \\
121.628\end{array}$ & (4) & (2) & $\begin{array}{c}153 \\
243 \\
063 \\
333 \\
U p \\
063 \\
333 \\
U_{p} \\
063 \\
333 \\
U p \\
063\end{array}$ & $\begin{array}{l}.04 \\
.04 \\
.05 \\
.03 \\
.03 \\
.03 \\
.04 \\
.04 \\
.09 \\
.06 \\
.04 \\
.07\end{array}$ & $\begin{array}{c}--- \\
--- \\
--- \\
--- \\
-- \\
--- \\
--- \\
-- \\
-- \\
-- \\
--- \\
---\end{array}$ \\
\hline
\end{tabular}

Note: One additional record ${ }^{1}$ recovered at Anderson Dam Structure Array.

Hollister

City Hall Annex

Basement (USGS)

Hollister Airport

Differential Array

(USGS)

18 October 1989

0008 G.m.t.

Central Calif.

Epicenter and

magnitude unknown
Anderson Dam

Morgan Hill

(USGS)

Structure Array:

$$
\text { Ch. 1-12 }
$$

Anderson Dam

Morgan Hill

(USGS)

Downstream

Structure Array:

Ch. 1-12 $\begin{array}{lll}36.851 & 07: 55.25 & 1.5\end{array}$

121.402

36.888

121.413

37.166

121.628

(4)

(2)

07:54.5 $\quad 1.1$

18 October 1989

Central Calif.

$37.198 \mathrm{~N}, 122.105 \mathrm{~W}$

Magnitude 5.1 ML 
Table 1. National Cooperative Strong-Motion Network Accelerograph Records Recovered During 1989-Continued

\begin{tabular}{l} 
Earthquake \\
\hline 18 October 1989 \\
0041:24.7 G.m.t. \\
Central Calif. \\
37.198N, 122.105W \\
Magnitude 5.1 ML \\
(Continued)
\end{tabular}

\section{Station Name}

(Owner)

$\begin{array}{lccccc}\begin{array}{l}\text { Coordinates } \\ \text { (Lat. }{ }^{\circ} \mathrm{N}\end{array} & \begin{array}{c}\text { Trigger } \\ \text { time }\end{array} & \begin{array}{c}\text { S-minus } \\ \text { trigger } \\ \left.\text { Long. }{ }^{\circ} \mathrm{W}\right)\end{array} & \begin{array}{c}\text { Direction } \\ (\mathrm{az})\end{array} & \begin{array}{c}\text { Maximum } \\ \text { amplitude }\end{array} & \begin{array}{c}\text { Duration } \\ (\mathrm{s})\end{array} \\ & & (\mathrm{s}) & & (\mathrm{g}) & \end{array}$

(2)

(1)

41.31.0
Rivera St.

(USGS)

\subsection{7}

121.879
18 October 1989

0323:57.0 G.m.t.

Central Calif.

$37.163 \mathrm{~N}, 121.995 \mathrm{~W}$

Magnitude 4.0 ML

18 October 1989

0518:34.1 G.m.t.

Central Calif.

$36.980 \mathrm{~N}, 121.847 \mathrm{~W}$

Magnitude 4.2 ML

18 October 1989

0639:10.1 G.m.t.

Central Calif.

$36.932 \mathrm{~N}, 121.712 \mathrm{~W}$

Magnitude 4.3 ML $\begin{array}{lr}\text { Anderson Dam } & 37.166 \\ \text { Morgan Hill } & 121.628\end{array}$

(USGS)

Structure Array

Ch. 1-12

Anderson Dam

Morgan Hill

(USGS)

Structure Array:

Ch. 1-12

Hollister

City Hall Annex

Basement (USGS)

Hollister Airport

Differential Array

(USGS)

18 October 1989-

19 October 1989

Central Calif.

Epicenters and

magnitudes unknown
Anderson Dam

Morgan Hill

(USGS)

Crest
(1)

37.166

(1)

$36.851 \quad 39: 16.6 \quad 5.3$

121.402

36.888

121.413

37.166

121.628

(3)

(2)

Note: Five additional records ${ }^{1}$ recovered at Anderson Dam Crest.

19 October 1989 1014:35.1 G.m.t. Central Calif. $36.963 \mathrm{~N}, 121.843 \mathrm{~W}$

Magnitude 4.6 ML

Bear Valley Station 14 Upper Butts Ranch (USGS)

\subsection{9}

121.043
$15: 04.8$

(2)

(1)
(1) 
Table 1. National Cooperative Strong-Motion Network Accelerograph Records Recovered During 1989-Continued

\begin{tabular}{|c|c|c|c|c|c|c|c|}
\hline Earthquake & $\begin{array}{l}\text { Station Name } \\
\text { (Owner) }\end{array}$ & $\begin{array}{l}\text { Coordinates } \\
\text { (Lat. }{ }^{\circ} \mathrm{N} \\
\text { Long. }{ }^{\circ} \mathrm{W} \text { ) }\end{array}$ & $\begin{array}{l}\text { Trigger } \\
\text { time }\end{array}$ & $\begin{array}{l}\text { S-minus } \\
\text { trigger } \\
\text { (s) }\end{array}$ & $\begin{array}{l}\text { Direction } \\
\text { (az) }\end{array}$ & $\begin{array}{l}\text { Maximum } \\
\text { amplitude } \\
\text { (g) }\end{array}$ & $\begin{array}{l}\text { Duration } \\
\text { (s) }\end{array}$ \\
\hline \multirow[t]{2}{*}{$\begin{array}{l}21 \text { October } 1989 \\
\text { 2214:57.0 G.m.t. } \\
\text { Central Calif. } \\
\text { 37.057N, 121.905W } \\
\text { Magnitude 4.9 ML }\end{array}$} & $\begin{array}{l}\text { Anderson Dam } \\
\text { Morgan Hill } \\
\text { (USGS) } \\
\text { Downstream }\end{array}$ & $\begin{array}{r}37.166 \\
121.628\end{array}$ & $15: 05.5$ & 0.3 & & (1) & \\
\hline & Crest & & & & $\begin{array}{l}340 \\
\text { Up } \\
250\end{array}$ & $\begin{array}{l}.03 \\
.03 \\
.06\end{array}$ & $\begin{array}{l}\cdots \\
--- \\
\cdots\end{array}$ \\
\hline \multirow[t]{2}{*}{$\begin{array}{l}25 \text { October } 1989 \\
0127: 26.6 \text { G.m.t. } \\
\text { Central Calif. } \\
37.078 \mathrm{~N}, 121.832 \mathrm{~W} \\
\text { Magnitude } 5.0 \mathrm{ML}\end{array}$} & $\begin{array}{l}\text { Anderson Dam } \\
\text { Morgan Hill } \\
\text { (USGS) }\end{array}$ & $\begin{array}{r}37.166 \\
121.628\end{array}$ & 27:34.1 & (2) & & & \\
\hline & $\begin{array}{l}\text { Downstream } \\
\text { Crest }\end{array}$ & & & & & $\begin{array}{l}\text { (1) } \\
\text { (1) }\end{array}$ & \\
\hline $\begin{array}{l}31 \text { October } 1989 \\
1905 \text { G.m.t. } \\
\text { Central Calif. } \\
\text { Epicenter and } \\
\text { magnitude unknown }\end{array}$ & $\begin{array}{l}\text { San Jose, } 101 / 280 / 680 \\
\text { Freeway Interchange } \\
\text { (USGS) }\end{array}$ & $\begin{array}{r}37.340 \\
121.851\end{array}$ & $05: 17.6$ & (2) & & (1) & \\
\hline $\begin{array}{l}3 \text { November } 1989 \\
\text { 1604:49.2 G.m.t. } \\
\text { Central Calif. } \\
36.512 \mathrm{~N}, 121.120 \mathrm{~W} \\
\text { Magnitude } 2.5 \mathrm{ML}\end{array}$ & $\begin{array}{l}\text { Bear Valley Station } 6 \\
\text { James Ranch } \\
\text { (USGS) }\end{array}$ & $\begin{array}{r}36.504 \\
121.101\end{array}$ & $04: 50.7$ & (2) & & (1) & \\
\hline $\begin{array}{l}\text { 7 November } 1989 \\
\text { 2342:37.7 G.m.t. } \\
\text { Central Calif. } \\
\text { 37.227N, 122.037W }\end{array}$ & $\begin{array}{l}\text { Cupertino } \\
\text { Pichetti Winery } \\
\text { (USGS) Temporary }\end{array}$ & $\begin{array}{r}37.294 \\
122.089\end{array}$ & (4) & (2) & & (1) & \\
\hline Magnitude 4.3 ML & $\begin{array}{l}\text { Los Gatos, Los Altos } \\
\text { Rod \& Gun Club } \\
\text { (USGS) Temporary }\end{array}$ & $\begin{array}{r}37.239 \\
122.106\end{array}$ & (4) & 1.6 & & (1) & \\
\hline $\begin{array}{l}10 \text { November } 1989 \\
1718 \text { G.m.t. } \\
\text { Central Calif. } \\
\text { Epicenter and } \\
\text { magnitude unknown }\end{array}$ & $\begin{array}{l}\text { San Jose, } 101 / 280 / 680 \\
\text { Freeway Interchange } \\
\text { (USGS/CDOT) }\end{array}$ & $\begin{array}{r}37.340 \\
121.851\end{array}$ & (4) & (2) & & (1) & \\
\hline $\begin{array}{l}10 \text { November } 1989 \\
2002 \text { G.m.t. } \\
\text { Central Calif. } \\
\text { Epicenter and }\end{array}$ & $\begin{array}{l}\text { Cupertino } \\
\text { Pichetti Winery } \\
\text { (USGS) Temporary }\end{array}$ & $\begin{array}{r}37.294 \\
122.089\end{array}$ & (4) & (2) & & (1) & \\
\hline magnitude unknown & $\begin{array}{l}\text { Los Gatos, Los Altos } \\
\text { Rod \& Gun Club } \\
\text { (USGS) Temporary }\end{array}$ & $\begin{array}{r}37.239 \\
122.106\end{array}$ & (4) & 1.6 & & (1) & \\
\hline
\end{tabular}


Table 1. National Cooperative Strong-Motion Network Accelerograph Records Recovered During 1989-Continued

\begin{tabular}{|c|c|c|c|c|c|c|c|}
\hline Earthquake & $\begin{array}{l}\text { Station Name } \\
\text { (Owner) }\end{array}$ & $\begin{array}{l}\text { Coordinates } \\
\text { (Lat. }{ }^{\circ} \mathrm{N} \\
\text { Long. }^{\circ} \mathrm{W} \text { ) }\end{array}$ & $\begin{array}{l}\text { Trigger } \\
\text { time }\end{array}$ & $\begin{array}{l}\text { S-minus } \\
\text { trigger } \\
\text { (s) }\end{array}$ & $\begin{array}{l}\text { Direction } \\
\quad \text { (az) }\end{array}$ & $\begin{array}{l}\text { Maximum } \\
\text { amplitude } \\
\text { (g) }\end{array}$ & $\begin{array}{l}\text { Duration } \\
\text { (s) }\end{array}$ \\
\hline
\end{tabular}

18 October 1989-

16 November 1989

Central Calif.

Epicenters and

magnitudes unknown
Palo Alto

VA Hospital Bldg. 1

(VA)

37.40

122.14

Basement

Roof (7th level)
(3)

(2)

Note: Two additional records ${ }^{1}$ recovered at Palo Alto VA Hospital roof level.

19 November 1989

0128 G.m.t.

Central Calif.

Epicenter and

magnitude unknown

20 November 1989

1339 G.m.t.

Central Calif.

Epicenter and

magnitude unknown

25 October 1989-

21 November 1989

Central Calif.

Epicenters and

Magnitudes unknown
Bear Valley Station 10

Webb Residence

(USGS)

San Jose, 101/280/680

Freeway Interchange

(USGS/CDOT)

37.340

121.851

Anderson Dam

Morgan Hill

(USGS)

Downstream

Crest $\begin{array}{lll}36.532 & 28: 59.7 & 1.5\end{array}$

121.143

(1)

(1)

(1)

37.166
121.628

340

.02

Up

250

.02

.06

Note: One additional record ${ }^{1}$ recovered at Anderson Dam crest.

Structure Array:

$\begin{array}{lcc}\text { Ch. 1- Mid-dam, Center } & 153 & .03 \\ \text { Ch. 2- Mid-dam, Center } & 243 & .04 \\ \text { Ch. 3- Mid-dam, Right } & 063 & .04 \\ \text { Ch. 4- Toe } & 333 & .03 \\ \text { Ch. 5- Toe } & \mathrm{Up} & .02 \\ \text { Ch. 6- Toe } & 063 & .03 \\ \text { Ch. 7- Right Crest } & 333 & .05 \\ \text { Ch. 8- Right Crest } & \mathrm{Up} & .04 \\ \text { Ch. 9- Right Crest } & 063 & .06 \\ \text { Ch. 10- Center Crest } & 333 & .03 \\ \text { Ch. 11- Center Crest } & \mathrm{Up} & .04 \\ \text { Ch. 12- Center Crest } & 063 & .06\end{array}$

Note: Three additional records ${ }^{1}$ recovered at Anderson Dam structure array. 
Table 1. National Cooperative Strong-Motion Network Accelerograph Records Recovered During 1989-Continued

\begin{tabular}{|c|c|c|c|c|c|c|c|}
\hline Earthquake & $\begin{array}{l}\text { Station Name } \\
\text { (Owner) }\end{array}$ & $\begin{array}{l}\text { Coordinates } \\
\text { (Lat. }{ }^{\circ} \mathrm{N} \\
\text { Long. }{ }^{\circ} \mathrm{W} \text { ) }\end{array}$ & $\begin{array}{l}\text { Trigger } \\
\text { time }\end{array}$ & $\begin{array}{l}\text { S-minus } \\
\text { trigger } \\
\text { (s) }\end{array}$ & $\begin{array}{l}\text { Direction } \\
\quad(a z)\end{array}$ & $\begin{array}{l}\text { Maximum } \\
\text { amplitude } \\
\text { (g) }\end{array}$ & $\begin{array}{l}\text { Duration } \\
\text { (s) }\end{array}$ \\
\hline
\end{tabular}

24 November 1989
1445:14.6 G.m.t.
Eastern Calif.
37.402N, 118.630W
Magnitude 3.9 ML

29 November 1989

1645:13.8 G.m.t.

Eastern Calif.

$37.520 \mathrm{~N}, 118.768 \mathrm{~W}$

Magnitude 3.0 ML

$\begin{array}{lr}\text { Chalfant Valley Array } & 37.53 \\ \text { Fire Station } & 118.37\end{array}$

(USGS)

McGee Creek

Mammoth Lakes

(USGS) SMA-1

McGee Creek

(USGS) CRA-1

$166 \mathrm{~m}$ Downhole

$35 \mathrm{~m}$ Downhole

Surface

$1 \mathrm{~m}$ Downhole
Mammoth Lakes

(1)

$37.550 \quad$ (4) (2)

(1)

118.811

37.550

118.811

(4)

(2)

(1)

(1)

(1)

(1)

21 October 1988-

1 December 1989

Southern Calif.

Epicenters and

magnitudes unknown

Los Angeles

34.071

444 S. San Vicente Blvd. $\quad 118.374$

(OWNR)

(3)

Roof (12th)

(2)

(1)

Note: Three additional records ${ }^{1}$ recovered at 444 S. San Vicente Boulevard roof.

5 December 1988-

1 December 1989

Southern Calif.

Epicenters and

magnitudes unknown Roof (8th)

Los Angeles

34.098

118.294

1526 N. Edgemont St.

(OWNR)

(3)
.08
.05
.13

$2.9 \quad 090 \quad .15$

Up $\quad .04$

$37.588 \mathrm{~N}$

118.705W

Crowley Lake

(USGS)

Left abutment
(1) 
Table 1. National Cooperative Strong-Motion Network Accelerograph Records Recovered During 1989-Continued

\begin{tabular}{|c|c|c|c|c|c|c|c|}
\hline Earthquake & $\begin{array}{l}\text { Station Name } \\
\text { (Owner) }\end{array}$ & $\begin{array}{l}\text { Coordinates } \\
\text { (Lat. }{ }^{\circ} \mathrm{N} \\
\text { Long. }^{\circ} \mathrm{W} \text { ) }\end{array}$ & $\begin{array}{c}\text { Trigger } \\
\text { time }\end{array}$ & $\begin{array}{l}\text { S-minus } \\
\text { trigger } \\
\text { (s) }\end{array}$ & $\begin{array}{l}\text { Direction } \\
\quad(a z)\end{array}$ & $\begin{array}{l}\text { Maximum } \\
\text { amplitude } \\
\text { (g) }\end{array}$ & $\begin{array}{l}\text { Duration } \\
\text { (s) }\end{array}$ \\
\hline $\begin{array}{l}14 \text { December } 1988- \\
1 \text { December } 1989 \\
\text { Southern Calif. } \\
\text { Epicenters and } \\
\text { magnitudes unknown }\end{array}$ & $\begin{array}{l}\text { Los Angeles } \\
2005 \text { N. Highland Ave. } \\
\text { (OWNR) } \\
\quad \text { Roof (8th) }\end{array}$ & $\begin{array}{c}34.106 \mathrm{~N} \\
118.336 \mathrm{~W}\end{array}$ & (3) & & & (1) & \\
\hline
\end{tabular}

Note: One additional record ${ }^{1}$ recovered at 2005 N. Highland Avenue roof.

\begin{tabular}{|c|c|c|c|c|c|c|c|}
\hline 2 December 1989 & Anza Array & 33.616 & (3) & 2.0 & 010 & .05 & --- \\
\hline 2316:47.8 G.m.t. & Garner Valley & 116.627 & & & Up & .05 & --- \\
\hline Southern Calif. & Fire Station (USGS) & & & & 280 & .04 & -- \\
\hline Magnitude 4.2 ML & Anza Array & 33.676 & $16: 50.6$ & 1.7 & 135 & .07 & --- \\
\hline & Hurkey Creek Park & 116.680 & & & Up & .07 & --- \\
\hline & (USGS) & & & & 045 & .08 & --- \\
\hline & Anza Array & 33.714 & (3) & 1.8 & 180 & .16 & 0.2 \\
\hline & Keenwild Forest Sta. & 116.711 & & & Up & .19 & 0.1 \\
\hline & (USGS) & & & & 090 & .18 & 0.2 \\
\hline & Anza Array & 33.61 & (3) & 3.2 & & (1) & \\
\hline & $\begin{array}{l}\text { Pinyon Flat Observ. } \\
\text { (USGS) }\end{array}$ & 116.46 & & & & & \\
\hline & Anza Array & 33.630 & $16: 53.2$ & (2) & 360 & .08 & -- \\
\hline & Red Mountain & 116.847 & & & Up & .02 & --- \\
\hline & (USGS) & & & & 270 & .06 & -- \\
\hline & Anza Array & 33.60 & (3) & 1.7 & 360 & .07 & --- \\
\hline & Tripp Flats & 116.74 & & & Up & .03 & --- \\
\hline & (USGS) & & & & 270 & .04 & --- \\
\hline & Anza Array & 33.578 & $16: 51.5$ & 2.6 & 360 & .05 & -- \\
\hline & Pine Meadow Ranch & 116.589 & & & Up & .03 & --- \\
\hline & (USGS) & & & & 270 & .05 & -- \\
\hline 17 August 1988- & Los Angeles & 34.058 & (3) & & & & \\
\hline 4 December 1989 & 2049 Century Park E. & 118.412 & & & & & \\
\hline & (OWNR) & & & & & & \\
\hline magnitudes unknown & 43rd floor & & & 4.9 & 320 & .05 & -- \\
\hline & & & & & Up & .07 & $-\cdots$ \\
\hline & & & & & 230 & .03 & \\
\hline
\end{tabular}


Table 1. National Cooperative Strong-Motion Network Accelerograph Records Recovered During 1989-Continued

\begin{tabular}{|c|c|c|c|c|c|c|c|}
\hline Earthquake & $\begin{array}{l}\text { Station Name } \\
\text { (Owner) }\end{array}$ & $\begin{array}{l}\text { Coordinates } \\
\text { (Lat. }{ }^{\circ} \mathrm{N} \\
\text { Long. }^{\circ} \mathrm{W} \text { ) }\end{array}$ & $\begin{array}{l}\text { Trigger } \\
\text { time }\end{array}$ & $\begin{array}{l}\text { S-minus } \\
\text { trigger } \\
\text { (s) }\end{array}$ & $\begin{array}{l}\text { Direction } \\
\text { (az) }\end{array}$ & $\begin{array}{l}\text { Maximum } \\
\text { amplitude } \\
\text { (g) }\end{array}$ & $\begin{array}{l}\text { Duration } \\
\text { (s) }\end{array}$ \\
\hline
\end{tabular}

17 August 1988-

4 December 1989

Southern Calif.

Epicenters and

magnitudes unknown 43rd floor

(Continued)

30 July $1987-$

5 December 1989

Southern Calif.

Epicenters and

magnitudes unknown

(OWNR)

Los Angeles

(OWNR)

10th fldor
Los Angeles

$\quad 118.413$

19191 S. Vermont Ave.

33.855

118.291
(3)

$\begin{array}{lll}4.8 & 320 & .06 \\ & \text { Up } & .09 \\ 230 & .02\end{array}$

(3)

$\begin{array}{ccc}4.3 & 360 & .09 \\ & \text { Up } & .09 \\ & 270 & .07 \\ & & \\ 10.8 & 360 & .06 \\ & \text { Up } & .03 \\ & 270 & .07\end{array}$

Note: Six additional records ${ }^{1}$ recovered at 19191 S. Vermont Avenue 10th floor.

3 August 1988-

6 December 1989

Southern Calif.

Epicenters and

magnitudes unknown
Los Angeles

5250 Century Blvd.

(OWNR)

Roof (8th)
33.945

118.372

(3) 
Table 1. National Cooperative Strong-Motion Network Accelerograph Records Recovered During 1989-Continued

\begin{tabular}{|c|c|c|c|c|c|c|c|}
\hline Earthquake & $\begin{array}{l}\text { Station Name } \\
\text { (Owner) }\end{array}$ & $\begin{array}{l}\text { Coordinates } \\
\text { (Lat. }{ }^{\circ} \mathrm{N} \\
\text { Long. }{ }^{\circ} \mathrm{W} \text { ) }\end{array}$ & $\begin{array}{l}\text { Trigger } \\
\text { time }\end{array}$ & $\begin{array}{l}\text { S-minus } \\
\text { trigger } \\
\text { (s) }\end{array}$ & $\begin{array}{l}\text { Direction } \\
\text { (az) }\end{array}$ & $\begin{array}{l}\text { Maximum } \\
\text { amplitude } \\
\text { (g) }\end{array}$ & $\begin{array}{l}\text { Duration } \\
\text { (s) }\end{array}$ \\
\hline
\end{tabular}

7 July 1988-

12 December 1989

Southern Calif.

Epicenters and

magnitudes unknown
Los Angeles

4929 Wilshire Blvd.

(OWNR)

Roof (11th)
34.063

118.337

(3)

$\begin{array}{lll}3.6 & 180 & .11 \\ & \text { Up } & .08 \\ & 090 & .07 \\ & & \\ 3.0 & 180 & .06 \\ & \text { Up } & .08 \\ & 090 & .06 \\ & & \\ 3.1 & 180 & .07 \\ & \text { Up } & .10 \\ & 090 & .05\end{array}$

1.5

$--$

Note: Two additional records ${ }^{1}$ recovered at 4929 Wilshire Boulevard roof.

17 August 198812 December 1989 Southern Calif. Epicenters and magnitudes unknown
Los Angeles

10100 Santa Monica Blvd. $\quad 118.416$ (OWNR)

Roof (27th)
(3) (s)

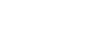


Table 1. National Cooperative Strong-Motion Network Accelerograph Records Recovered During 1989-Continued

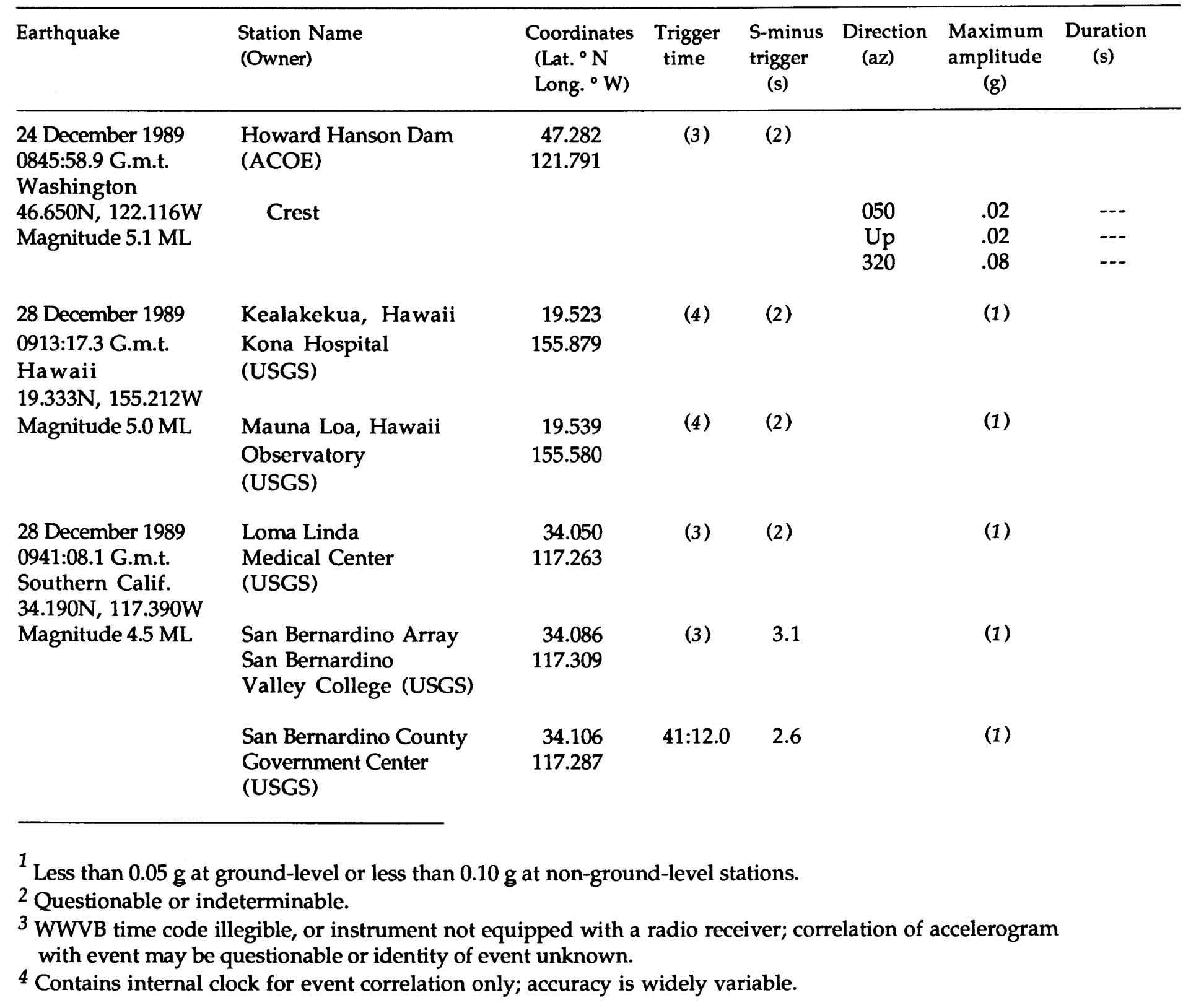





\section{SELECTED SERIES OF U.S. GEOLOGICAL SURVEY PUBLICATIONS}

\section{Perlodicals}

Earthquakes \& Volcanoes (issued bimonthly).

Preliminary Determination of Epicenters (issued monthly).

\section{Technical Books and Reports}

Professional Papers are mainly comprehensive scientific reports of wide and lasting interest and importance to professional scientists and engineers. Included are reports on the results of resource studies and of topographic, hydrologic, and geologic investigations. They also include collections of related papers addressing different aspects of a single scientific topic.

Bulletins contain significant data and interpretations that are of lasting scientific interest but are generally more limited in scope or geographic coverage than Professional Papers. They include the results of resource studies and of geologic and topographic investigations; as well as collections of short papers related to a specific topic.

Water-Supply Papers are comprehensive reports that present significant interpretive results of hydrologic investigations of wide interest to professional geologists, hydrologists, and engineers. The series covers investigations in all phases of hydrology, including hydrogeology, availability of water, quality of water, and use of water.

Circulars present administrative information or important scientific information of wide popular interest in a format designed for distribution at no cost to the public. Information is usually of short-term interest.

Water-Resources Investigations Reports are papers of an interpretive nature made available to the public outside the formal USGS publications series. Copies are reproduced on request unlike formal USGS publications, and they are also available for public inspection at depositories indicated in USGS catalogs.

Open-File Reports include unpublished manuscript reports, maps, and other material that are made available for public consultation at depositories. They are a nonpermanent form of publication that may be cited in other publications as sources of information.

\section{Maps}

Geologic Quadrangle Maps are multicolor geologic maps on topographic bases in $71 / 2$-or 15 -minute quadrangle formats (scales mainly $1: 24,000$ or $1: 62,500$ ) showing bedrock, surficial, or engineering geology. Maps generally include brief texts; some maps include structure and columnar sections only.

Geophysical Investigations Maps are on topographic or planimetric bases at various scales; they show results of surveys using geophysical techniques, such as gravity, magnetic, seismic, or radioactivity, which reflect subsurface structures that are of economic or geologic significance. Many maps include correlations with the geology.

Miscellaneous Investigations Series Maps are on planimetric or topographic bases of regular and irregular areas at various scales; they present a wide variety of format and subject matter. The series also includes 7 1/2-minute quadrangle photogeologic maps on planimetric bases which show geology as interpreted from aerial photographs. Series also includes maps of Mars and the Moon.
Coal Investigations Maps are geologic maps on topographic or planimetric bases at various scales showing bedrock or surficial geology, stratigraphy, and structural relations in certain coal-resource areas.

Oil and Gas Investigations Charts show stratigraphic information for certain oil and gas fields and other areas having petroleum potential.

Miscellaneous Field Studies Maps are multicolor or black-andwhite maps on topographic or planimetric bases on quadrangle or irregular areas at various scales. Pre-1971 maps show bedrock geology in relation to specific mining or mineral-deposit problems; post-1971 maps are primarily black-and-white maps on various subjects such as environmental studies or wildemess mineral investigations.

Hydrologic Investigations Atlases are multicolored or black-andwhite maps on topographic or planimetric bases presenting a wide range of geohydrologic data of both regular and irregular areas; principal scale is $1: 24,000$ and regional studies are at $1: 250,000$ scale or smaller.

\section{Catalogs}

Permanent catalogs, as well as some others, giving comprehensive listings of U.S. Geological Survey publications are available under the conditions indicated below from the U.S. Geological Survey, Books and Open-File Reports Section, Federal Center, Box 25425, Denver, CO 80225. (See latest Price and Availability List.)

"Publications of the Geological Survey, 1879-1961" may be purchased by mail and over the counter in paperback book form and as a set of microfiche.

"Publications of the Geological Survey, 1962-1970" may be purchased by mail and over the counter in paperback book form and as a set of microfiche.

"Publications of the U.S. Geological Survey, 1971- 1981" may be purchased by mail and over the counter in paperback book form (two volumes, publications listing and index) and as a set of microfiche.

Supplements for 1982, 1983, 1984, 1985, 1986, and for subsequent years since the last permanent catalog may be purchased by mail and over the counter in paperback book form.

State catalogs, "List of U.S. Geological Survey Geologic and Water-Supply Reports and Maps For (State)," may be purchased by mail and over the counter in paperback booklet form only.

"Price and Availability List of U.S. Geologlcal Survey Publications," issued annually, is available free of charge in paperback booklet form only.

Selected coples of a monthly catalog "New Publications of the U.S. Geological Survey" available free of charge by mail or may be obtained over the counter in paperback booklet form only. Those wishing a free subscription to the monthly catalog "New Publications of the U.S. Geological Survey" should write to the U.S. Geological Survey, 582 National Center, Reston, VA 22092.

Note.--Prices of Government publications listed in older catalogs, announcements, and publications may be incorrect. Therefore, the prices charged may differ from the prices in catalogs, announcements, and publications. 
